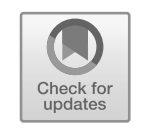

\title{
4 Aufgaben im Luzerner Modell
}

Zum Aufgabenprofil und zum Arbeitsalltag sozialpolitischer Verantwortungsträgerinnen und Verantwortungsträger gehört es unter anderem, neue soziale Dienstleistungen zu entwickeln, bestehende Angebote und Strukturen zu überprüfen und weiterzuentwickeln oder die Erbringung sozialer Dienstleistungen zu überwachen. In der Schweiz sind für diese Aufgaben mehrheitlich die Gemeinden und Kantone zuständig. Die kantonalen und kommunalen Verwaltungsabteilungen verfügen diesbezüglich über einen grossen Erfahrungsschatz und einschlägiges Fachwissen. Wie im vorhergehenden Kapitel deutlich gemacht wurde, haben sich in den letzten Jahrzehnten die politischen Legitimationsbedingungen und damit auch die fachlichen, betriebswirtschaftlichen und rechtlichen Ansprüche im Sozialbereich deutlich gesteigert. Interessierte Bürgerinnen und Bürger betrachten zunehmend genauer, wie sich die Kosten und die Leistungserbringung im Sozialbereich entwickeln. Trägerschaften und Führungspersonen sozialer Einrichtungen haben auf diese Umweltveränderungen mit einer Professionalisierung reagiert; der rasante Ausbau der Sozialmanagement-Lehrgänge zeugt davon (Boessenecker/Markert 2011). Ein systematischer Fach- und Methodendiskurs zur Gestaltung von ganzen Versorgungsbereichen oder Versorgungssystemen befindet sich indes erst in den Anfängen; dies gilt insbesondere für die Schweiz. In Deutschland hat die Professionalisierung dieser Aufgaben im Rahmen der Diskurse um Sozialplanung schon länger Fahrt aufgenommen und ist heute breiter etabliert. In Fachkreisen ist unbestritten, dass die verbesserte Planung und Abstimmung von Leistungen einzelner Organisationen und der Einbezug unterschiedlichster Akteure in (Versorgungs-) Netzwerken ein beachtliches Optimierungspotenzial birgt.

Dieses Kapitel verfolgt das Ziel, wichtige Aufgaben in der Systemgestaltung systematisch darzustellen. Das Zusammenführen einzelner Aufgaben zu einem Handlungsmodell möchte - wie schon das letzte Kapitel auf der Ebene des Handlungsverständnisses - einen Beitrag zur Professionalisierung in der Gestaltung von Versorgungssystemen leisten. Damit ein Handlungsmodell seinen Namen verdient, sollte das Modell unter anderem zwei Kriterien genügen: erstens für die Bewältigung konkreter Anwendungs- und alltäglicher Praxisfragen dienlich zu sein (Handlungsbezug) und zweitens eine gewisse systematische und nachvollziehbare Reflexions- oder Arbeitsweise zu ermöglichen (Modellbezug).

Um den Handlungsbezug darzulegen, wird in den folgenden Abschnitten bei der Präsentation jeder einzelnen der insgesamt 16 Gestaltungsaufgaben in der Regel ein Illustrationsbeispiel angefügt. Anschliessend wird im Kapitel 5 ferner an 
drei Praxisbeispielen deutlich gemacht, wie das LUS in unterschiedlichen Kontexten angewendet werden kann. Es handelt sich um:

- die Umsetzung der NFA im Bereich der Behindertenhilfe im Kanton Zug ( $A b$ schnitt 5.1);

- die Überprüfung und Konzeptualisierung der Basis-, Beschäftigungs- und Integrationsprogramme (BBIP) für Flüchtlinge und vorläufig Aufgenommene im Kanton Zürich (Abschnitt 5.2);

- das Versorgungskonzept «Gesundheit und Alter» der Gemeinde Kriens (Abschnitt 5.3).

Dabei wird das Handlungsmodell in allen drei Fällen im Nachhinein einem bereits umgesetzten und dokumentierten, anspruchsvollen Planungs- und Gestaltungprozess gegenübergestellt. In unterschiedlicher Ausprägung können damit in allen drei Beispielen die Phasen des LUS sichtbar gemacht werden. Um den Bezug zwischen den Arbeitsschritten des LUS und einer praktischen Anwendung zu verdeutlichen, wird in den folgenden Abschnitten - wann immer sinnvoll - auf ausgewählte Aspekte der Praxisbeispiele des fünften Kapitels verwiesen.

Im folgenden Abschnitt werden die Bezugspunkte dargestellt, die zur Eruierung und Systematisierung der 16 Gestaltungsaufgaben geführt haben. In den weiteren Abschnitten dieses Kapitels folgt anschliessend die Darstellung der einzelnen Gestaltungsaufgaben.

\subsection{Bezüge des Handlungsmodells zur Systemgestaltung}

\subsubsection{Erster Bezug: Vier Ebenen der Systemgestaltung}

Bei der Beschreibung des LUS für die Sozialwirtschaft im Abschnitt 2.4 wurde dargelegt, dass die Systemgestaltung eine von vier zentralen Aufgaben darstellt. Der Systemgestaltung (Feld b in Abbildung 12) kommt zum einen die Aufgabe zu, relevante Themen des öffentlichen Diskurses über soziale Probleme (Feld a) aufgrund von fachlichen und / oder politischen Dringlichkeiten auszuwählen. Zum anderen gilt es, im Rahmen der Systemgestaltung die Leistungserbringung in einzelnen Einrichtungen (Feld c) sowie innerhalb von Versorgungssystemen (Feld d) zu planen und durchzuführen. 


\section{Diskurs soziale Probleme (a) \\ - Gesellschaftlicher Diskurs über soziale Probleme (Akteure, Medien, Themen usw.) \\ - Interessensvermittlung (Vernehmlassungen, Interes- sensorganisationen usw.)}

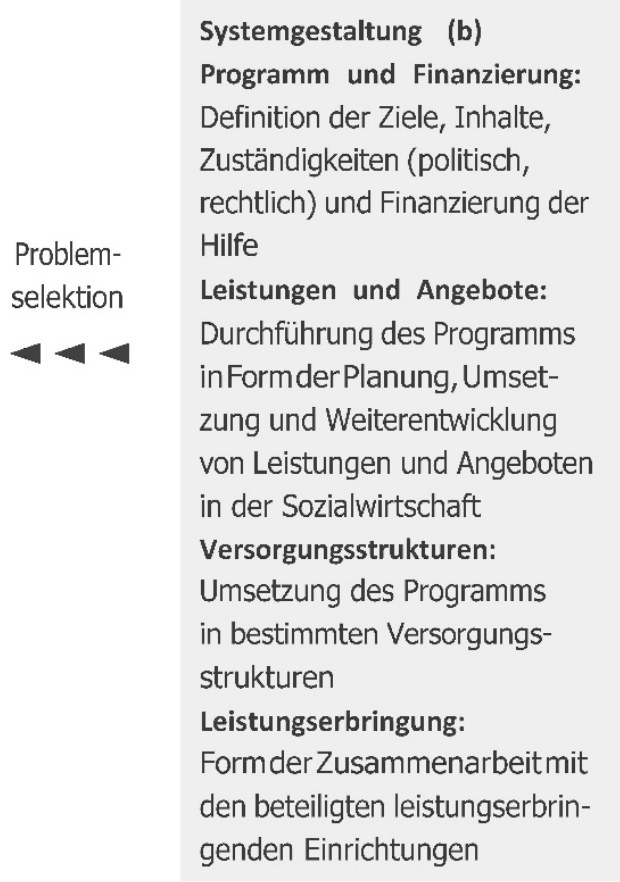

Leistungs-
vereinbarungen

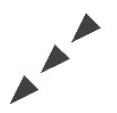

Systemgestaltung (b)

Programm und Finanzierung:

Definition der Ziele, Inhalte, Zuständigkeiten (politisch,

gebote: zung und Weiterentwicklung von Leistungen und Angeboten in der Sozialwirtschaft Versorgungsstrukturen Umsetzung des Programms in bestimmten Versorgungsstrukturen Leistungserbringung: Formder Zusammenarbeitmit genden Einrichtungen

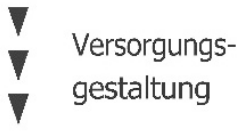

Versorgungssysteme (d)

Koordinationsfragen rechtlich) und Finanzierung der inFormderPlanung, Umset-

\section{Leistungserbringende}

\section{Organisation (c)}

Führung/Managementeiner sozialen Einrichtung

Abbildung 12: Ebenen der Systemgestaltung

Quelle: eigene Darstellung 
Wie in der Abbildung dargestellt, sind für die Systemgestaltung vier Ebenen relevant:

(1) die Entwicklung und Umsetzung von Programm und Finanzierung im Kontext politischer, finanzieller und rechtlicher Rahmenbedingungen;

(2) die Umsetzung des Hilfskonzepts in Leistungen und Angebote in der Sozialwirtschaft;

(3) die Entwicklung von Versorgungsstrukturen;

(4) die Leistungserbringung.

Die vier Gestaltungsebenen stellen den ersten Baustein des Handlungsmodells dar.

\subsubsection{Zweiter Bezug: Vier Phasen des Managementkreislaufs}

Da die Systemgestaltung managementbezogenen Handlungsweisen gleicht, richtet sich der zweite Baustein an den Phasen klassischer Managementkreisläufe aus. Wie die folgende Abbildung zeigt, wurde der Kreislauf in vier Phasen aufgeteilt:

(1) Analyse;

(2) Strategie;

(3) Umsetzung sowie

(4) Weiterentwicklung.

Im Folgenden wird von einem Gestaltungskreislauf respektive einem Kreislauf der Systemgestaltung gesprochen. 


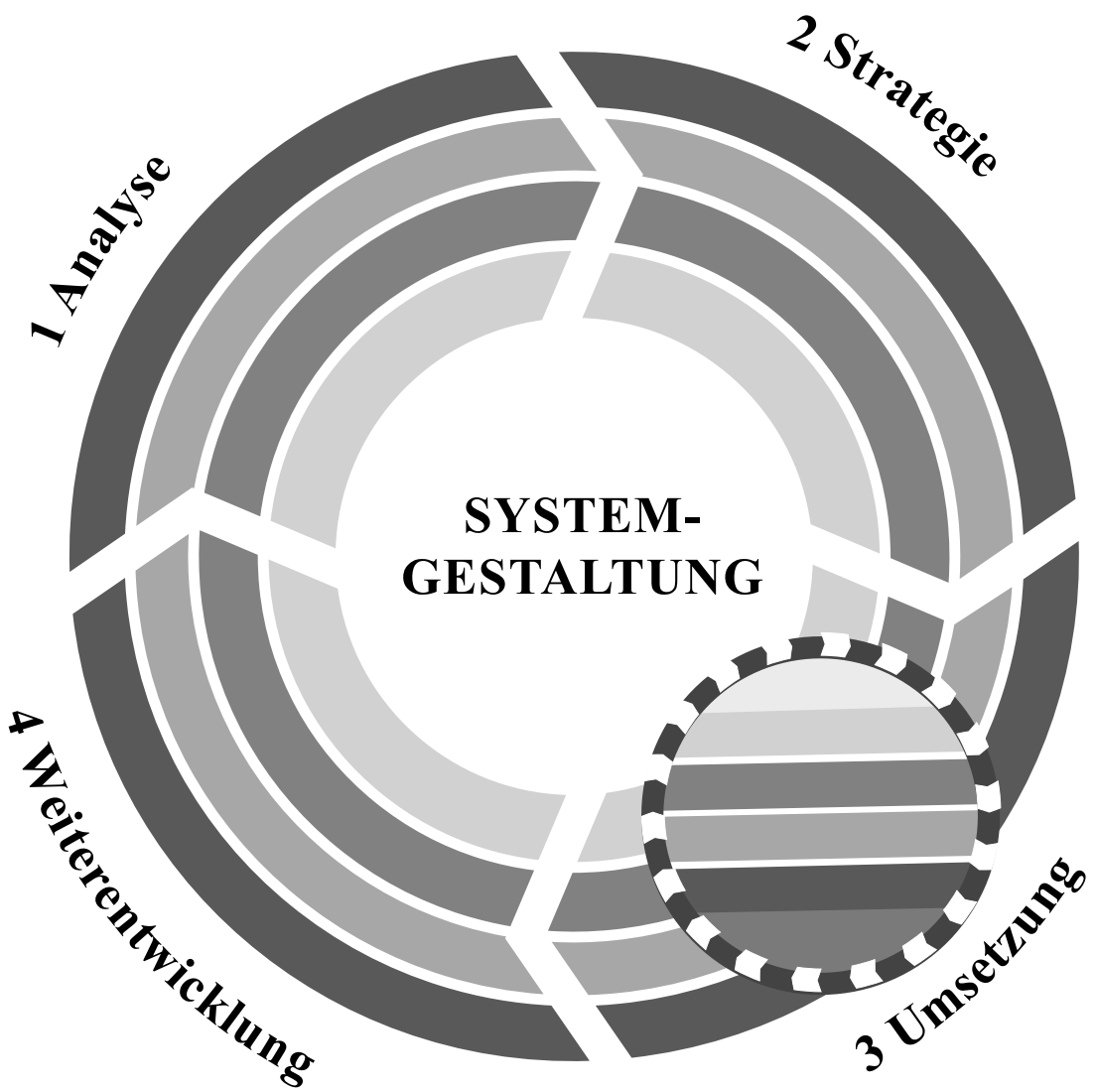

Abbildung 13: Gestaltungskreislauf Quelle: eigene Darstellung

Während die Phasen 'Analyse', 'Strategie' sowie 'Weiterentwicklung' in der Regel einem mehrjährigen Zyklus folgen, findet die operative Phase 'Umsetzung' laufend statt. Aus diesem Grund ist sie in der Abbildung gesondert dargestellt. 


\subsubsection{Grundzüge des Modells: 16 Aufgaben im Gestaltungskreislauf}

Die Kombination des ersten Bausteins 'Gestaltungsebenen' mit dem zweiten Baustein 'Phasen des Management- oder Gestaltungskreislaufs' erlaubt es für jede Phase, einzelne Aufgaben zu identifizieren. Pro Phase lassen sich dadurch vier zentrale Aufgaben spezifizieren. Wie die folgenden beiden Abbildungen zeigen, resultieren daraus im gesamten Gestaltungskreislauf insgesamt 16 Aufgaben.

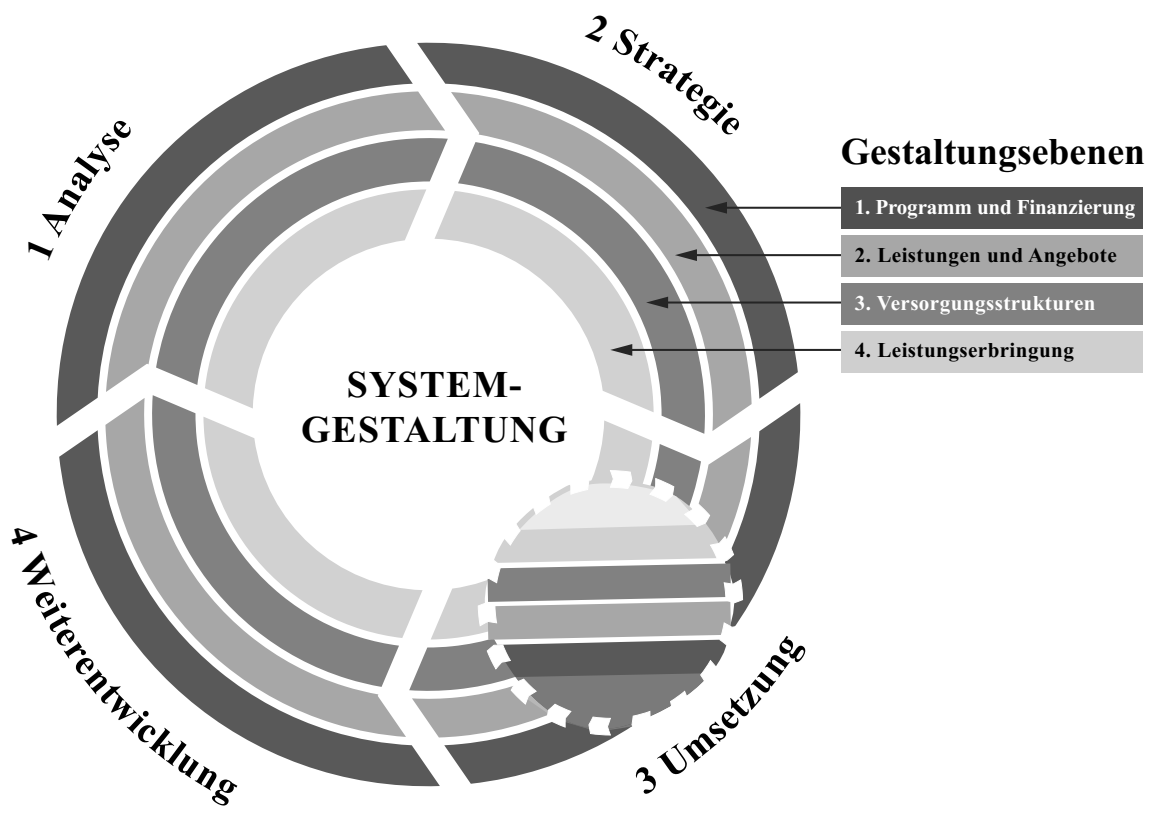

Abbildung 14: Gestaltungsebenen im Gestaltungskreislauf Quelle: eigene Darstellung 


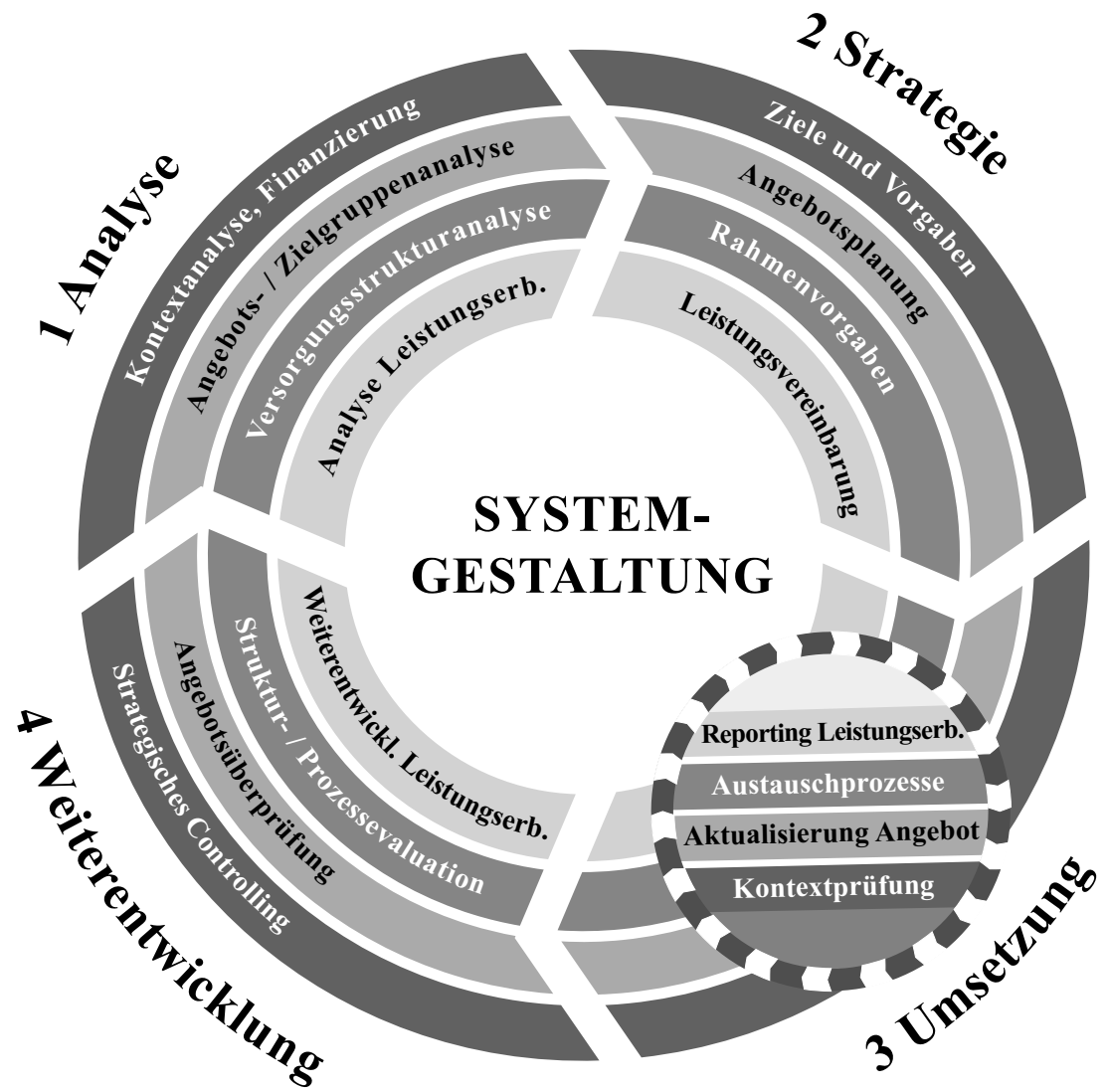

Abbildung 15: Übersicht zu den 16 Aufgaben im Gestaltungskreislauf Quelle: eigene Darstellung

In den folgenden Abschnitten werden die vier Aufgaben jeder Phase des Gestaltungskreislaufs kurz beschrieben und ihre Anwendung mit Beispielen illustriert. Die Illustrationsbeispiele entstammen der Alters- und der Flüchtlingspolitik und beleuchten in der Regel einzelne Aspekte der beschriebenen Gestaltungsaufgaben. Um die praktische Verwendbar- und Handhabbarkeit des Gestaltungsmodells vertiefter darzulegen, wird im fünften Kapitel an drei Praxisbeispielen deutlich gemacht, wie das LUS in unterschiedlichen Kontexten angewendet werden kann. 
Die drei Praxisbeispiele beziehen sich auf die kantonale Behindertenhilfe, Arbeitsintegrationsangebote für Flüchtlinge und vorläufig Aufgenommene sowie die kommunale Alterspolitik. In den nächsten Abschnitten wird oft auch Bezug auf die Praxisbeispiele im fünften Kapitel genommen.

\subsection{Gestaltungsphase 1: Analyse (A)}

Gestaltungsprozesse beginnen mit einer Analyse. Werden der Analysephase die vier definierten Gestaltungsebenen in Versorgungssystemen - 'Programm und Finanzierung' (1), 'Leistungen und Angebote' (2), 'Versorgungsstrukturen' (3) sowie 'Leistungserbringung' (4) - zugeordnet, ergeben sich folgende vier Gestaltungsaufgaben:

(A1) die Analyse von Kontextentwicklungen sowie die Prüfung des gesetzlichen und politischen Regelungs- und des Finanzierungsbedarfs und der Finanzierungsmöglichkeiten (Gestaltungsebene 1: 'Programm und Finanzierung');

(A2) die Durchführung von Angebots- und Zielgruppenanalysen (Gestaltungsebene 2: 'Leistungen und Angebote');

(A3) die Untersuchung von Versorgungsstrukturen (Gestaltungsebene 3: Versorgungsstrukturen);

(A4) die Analyse der Leistungserbringenden (Gestaltungsebene 4: 'Leistungserbringung'). 
Die folgende Abbildung gibt einen Überblick über die Aufgaben der Situationsanalyse.

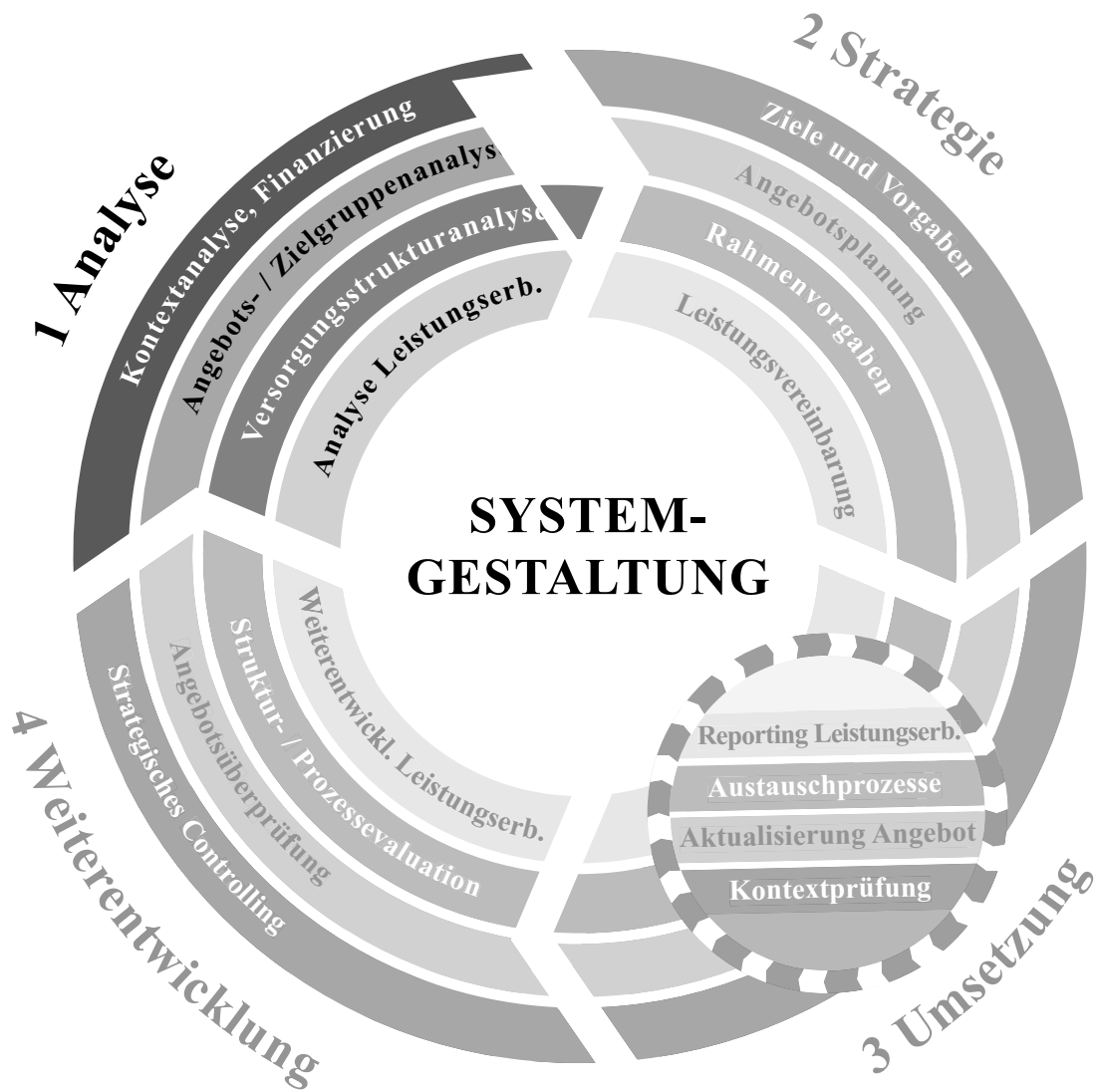

Abbildung 16: Analyseaufgaben in der Systemgestaltung: Phase 1 Quelle: eigene Darstellung

\subsubsection{Gestaltungsaufgabe A1: Kontextanalyse und Finanzierung}

Im Rahmen einer Situationsanalyse empfiehlt es sich auf der ersten Gestaltungsebene 'Programm und Finanzierung' und mit Blick auf mögliche Ziele, Angebote oder Dienstleistungen, eine Kontext-und Realisierbarkeitsanalyse durchzuführen. 
Die Analyse von Kontextentwicklungen dient primär der Einschätzung einer potenziellen Nachfrage oder der Begründung des Problemdrucks. Ebenfalls angezeigt ist eine Prüfung der Finanzierbarkeit, der politischen Realisierbarkeit und entsprechender gesetzlicher Rahmenbedingungen. Folgende Analysefragen stellen sich in diesem Zusammenhang:

- $\quad$ Rechtlicher Regelungsbedarf: Welche relevanten rechtlichen oder gesetzlichen Grundlagen existieren (z.B. im Gesetz definierte Aufgaben, bestimmte gesetzliche Zuständigkeiten)? Welcher rechtliche respektive gesetzliche Regelungsbedarf besteht (z.B. auf Verordnungsstufe)?

- $\quad$ Politischer Handlungsbedarf: Resultiert Regelungsbedarf aus entsprechenden politischen Vorstössen? Erhalten bestimmte politische Einheiten wie Kantone oder Gemeinden neue, zum Beispiel gesetzlich verankerte Aufträge?

- Finanzierungsbedarf und Finanzierungsmöglichkeiten: Wie stellt sich die Finanzierungssituation (Zuständigkeiten, gesetzliche Grundlagen, politische Vorgaben) dar? Welche finanziellen Möglichkeiten, Spielräume und Restriktionen bestehen? Welcher Finanzierungsbedarf für die Realisierung des Leistungsangebots wird veranschlagt? Welche Realisierungschancen existieren?

- Kontextentwicklungen: Welche gesellschaftlichen Trends oder Problembelastungen in der Umwelt des Versorgungsnetzes lassen sich beobachten? Welche dieser Trends sind in welcher Form relevant für die Nachfrage respektive die Angebotsentwicklung im Versorgungsnetz? Welche quantitativen und qualitativen Trends in der Nachfrage lassen sich mittel- oder längerfristig prognostizieren?

Zur Ermittlung wichtiger Kontextentwicklungen eignen sich klassische Literaturrecherchen und Datenanalysen. Hilfreich bei der Konsultation und Verarbeitung entsprechender Grundlagen ist die Unterscheidung zwischen quantitativen und qualitativen Aspekten, Entwicklungen oder Trends. Quantitative Aspekte geben primär Hinweise auf die mögliche zukünftige Nachfrage und die zahlenmässige Bedeutung der untersuchten Kontextentwicklungen. Qualitative Aspekte weisen auf den fachlichen Entwicklungs- oder Weiterentwicklungsbedarf hin und sind besonders relevant für die Formulierung und Festlegung der strategischen Ausrichtung (siehe zweite Phase des Gestaltungskreislaufs). Zur Ermittlung qualitativer Entwicklungen und Trends eignen sich insbesondere auch Expertenhearings oder Expertenbefragungen. Sie stellen in der Regel eine effiziente Art und Weise dar, wichtige Kontextentwicklungen und Trends in komprimierter Form in Erfahrung zu bringen. Mit Bezug auf den im dritten Kapitel erwähnten Grundsatz der Partizipation empfiehlt es sich im Rahmen von Kontextanalysen zudem, betroffene Be- 
völkerungsgruppen in die Situationsanalyse einzubeziehen; sei es durch mündliche oder schriftliche Befragungen, sei es durch öffentliche Diskussions- und Austauschanlässe (moderierte Grossgruppenanlässe, Zukunftswerkstätten usw.). Der systematische Einbezug von Betroffenen gewinnt zunehmend an Bedeutung, wie Konzepte der partizipativen Qualitätsentwicklung zeigen (Wright 2010).

\section{Beispiel - Entwicklungskonzept «Altern in Luzern»}

Das Entwicklungskonzept «Altern in Luzern», das im Oktober 2011 vom Parlament der Stadt Luzern verabschiedet wurde, beginnt mit einer Kontextanalyse und befasst sich dabei mit der demografischen Entwicklung sowie mit gesellschaftlichen, kulturellen und sozialen Aspekten des Alterns. Im Folgenden wird als Beispiel ein Auszug aus dem Bericht an das städtische Parlament (Stadt Luzern 2011) präsentiert.

Der Altersquotient (Anteil der über 65-jährigen an der ständigen Wohnbevölkerung) liege im gesamtschweizerischen Mittel bei $16.7 \%$. Im Stadtkreis Littau sei er mit 13.7\% tiefer und in der Stadt Luzern mit 21.5\% höher als die gesamtschweizerische Referenzgrösse. Die ungleiche Altersverteilung sei kein Spezifikum von Luzern. Der in den Städten tendenziell höhere Anteil älterer Menschen gegenüber den Agglomerationsgürteln liesse sich vielerorts feststellen.

\begin{tabular}{|c|c|c|c|c|}
\hline Stadt Luzern & Total & $\begin{array}{c}\text { 65 Jahre und } \\
\text { älter }\end{array}$ & $\begin{array}{c}\text { 80 Jahre und } \\
\text { älter }\end{array}$ & $\begin{array}{c}\text { 90 Jahre und } \\
\text { älter }\end{array}$ \\
\hline 2015 & $8^{\prime} 075(100 \%)$ & $1^{\prime} 633(18 \%)$ & $5^{\prime} 221(6 \%)$ & $1^{\prime} 069(1 \%)$ \\
\hline 2020 & $88^{\prime} 528(100 \%)$ & $1^{\prime} 943(18 \%)$ & $5^{\prime} 333(6 \%)$ & $1^{\prime} 912(1 \%)$ \\
\hline 2030 & $9^{\prime} 360(100 \%)$ & $1^{\prime} 7^{\prime} 445(18 \%)$ & $5^{\prime} 996(6 \%)$ & $1^{\prime} 368(1 \%)$ \\
\hline
\end{tabular}

Abbildung 17: Bevölkerungsszenarium Stadt Luzern 2015-2030

Quelle: LUSTAT Statistik Luzern; kantonale Bevölkerungsprognosen, Gesundheits- und Sozialdepartement, 2. Februar 2010

Aus gerontologischer Sicht sei vor allem bedeutsam, dass für Frauen und Männer die Lebenserwartung bei guter Gesundheit gestiegen ist. Allerdings bestünde betreffend die Lebenserwartung nach wie vor eine deutliche und sogar zunehmende Ungleichheit aufgrund des sozioökonomischen Status. Je höher der Bildungsstand sei, desto höher sei die Lebenserwartung. Dieser demografische Wandel, der auch für die Stadt Luzern eine Zunahme der älteren Wohnbevölkerung mit sich bringen werde, bringe auch eine nachhaltige Änderung in das Leben der Stadt Luzern. Die Alterspolitik, die bisher eher einer Versorgungspolitik zuzuordnen sei, müsse sich nebst diesem weiterzuverfolgenden Bereich auch noch in eine andere Richtung 
orientieren. Der Alterungsprozess der ganzen Bevölkerung werde eine gesellschaftspolitische Herausforderung sein, die den herkömmlichen Rahmen sprengen werde. In einer alternden Stadtgesellschaft rückten zusätzlich das Wohnumfeld, die Mobilität sowie die soziale Teilhabe in den Vordergrund.

In den weiteren Ausführungen werden im Entwicklungskonzept der Stadt Luzern die alterspolitischen Rahmenbedingungen auf nationaler, kantonaler und kommunaler Ebene analysiert. Die Analyse geht auf die alterspolitische Strategie des Bunds ein, nimmt das kantonale Altersleitbild in den Blick, betrachtet die bisherige städtische Alterspolitik und fasst die jüngsten parlamentarischen Forderungen nach einer neuen städtischen Alterspolitik zusammen. Aus all diesen Analysen werden der Handlungsbedarf und die Realisierungsbedingungen für die Weiterentwicklung der Alterspolitik in der Stadt Luzern hergeleitet.

Hinweise auf die Umsetzung der Gestaltungsaufgabe 'Kontextanalyse, Finanzierung und Realisierbarkeit' in den Anwendungsbeispielen

Übergabe der Behindertenhilfe vom Bund an die Kantone am Beispiel des Kantons Zug (Abschnitt 5.1):

Mittels Analysen qualitativer und quantitativer Daten wurde und wird im Kanton Zug versucht, periodisch folgende Fragen zu beantworten: Wie stellt sich die Situation von Menschen mit Behinderung in der hiesigen Gesellschaft dar und welche Entwicklungen sind absehbar? Welcher Bedarf an Einrichtungen und Leistungen für Menschen mit Behinderung ist heute feststellbar und wie wird sich dieser Bedarf entwickeln? Welche Einflussmöglichkeiten auf die Entwicklung des Bedarfs bestehen? Mit welchem Finanzbedarf ist zu rechnen?

Entwicklung des Versorgungskonzepts «Gesundheit und Alter» der Gemeinde Kriens (Abschnitt 5.3):

Mit Blick auf den Aufbau eines Versorgungssystems «Gesundheit und Alter» (Steuergruppe Versorgungskonzept «Gesundheit und Alter» Kriens 2011) wurde in Kriens eine breite, differenzierte und fachlich gut abgestützte Bestandsaufnahme realisiert. Dies zeigt sich in den Analysen zu Kontext- und Nachfrageentwicklungen in Form einer Prognose der Entwicklung der älteren Bevölkerung sowie des künftigen Pflegebedarfs und einer Analyse der politischen Rahmenbedingungen. ${ }^{12}$

12 Informationen zum Konzept sind zu finden unter: https://www.hslu.ch/de-ch/hochschuleluzern/forschung/projekte/detail/?pid=220 (24.01.2018). 


\subsubsection{Gestaltungsaufgabe A2: Angebots- und Zielgruppenanalyse}

Während sich Kontext- und Nachfrageanalysen sowie die generelle Überprüfung der rechtlichen, politischen und finanziellen Realisierbarkeit auf das Umfeld beziehen, sind Angebots- und Zielgruppenanalysen auf das Versorgungssystem selbst ausgerichtet. Die zweite Gestaltungsaufgabe stellt sozusagen das systeminterne Gegenstück zur Kontext- und Realisierungsanalyse dar. Daraus resultieren folgende Fragen für die Analyse:

- Angebotsinventar /-typologie: Welche Angebote werden in welcher Häufigkeit erbracht und wie lassen sie sich sinnvoll zu Angebotstypen zusammenfassen?

Für die Analyse (bestehender) Angebote eignen sich unter anderem sogenannte Angebotsinventare. Im Rahmen von Angebotsinventaren werden diejenigen Leistungen kategorisiert und statistisch erhoben, die für eine zukünftige Leistungs- und Angebotsgestaltung besonders relevant sind. In der Regel ist es zweckmässig, bestimmte Dienstleistungen zu Angebotspaketen zusammenzufassen und eine Typologie zu entwickeln, die zwischen fünf und höchstens zehn verschiedenen Angebotsbereichen oder Angebotsformen unterscheidet.

- Zielgruppenmerkmale: Welche Merkmale zeichnen die (potenziellen) Zielgruppen des Angebots aus? Wie lassen sich Typen von Zielgruppen bilden? Wegleitend für die Unterscheidung von Zielgruppen ist deren Betreuungs-, Unterstützungs- oder Beratungsbedarf. Angebotsinventare oder Zielgruppentypologien lassen sich durch eine regelmässige statistische Erhebung - zum Beispiel einmal im Jahr - zu sogenannten Angebots- oder Zielgruppenmonitorings ausbauen. Auf diese Weise können zentrale Eckdaten für die Systemgestaltung zur Verfügung gestellt werden.

- Angebotsanalyse: Wie gut decken die Angebote die Nachfrage ab (Angebotsdichte, Auslastung, Lücken)? Entspricht das Angebotsprofil den Zielgruppen?

\section{Beispiel - Zielgruppentypologie als Grundlage für die Angebotsanalyse}

Am 1. Januar 2008 gingen die Finanzierung und die Umsetzung der Integrationsmassnahmen für Flüchtlinge und vorläufig Aufgenommene vom Bund an die Kantone über. Ein Team der Hochschule Luzern unterstützte die Verantwortlichen des Kantons Zürich bei der Konzipierung und erstmaligen Umsetzung dieser Aufgaben. 
Die Angebotsanalyse zu Beginn des Projekts zeigte, dass ein zentraler Schwachpunkt in der kaum formalisierten Zieldefinition der meisten untersuchten Angebote lag. Ebenso mangelte es an formalisierten oder auf die Zielsetzung abgestimmten Instrumenten zur Überprüfung der Zielerreichung. Die Teilnehmenden waren in Bezug auf Schulgewohntheit und / oder Leistungsfähigkeit sehr heterogen, ohne dass mit ihnen unterschiedliche Ziele verfolgt worden wären. Sie behinderten sich in den Integrationsprogrammen zum Teil gegenseitig. Aus diesem Grund wurde eine Zielgruppentypologie entwickelt. Sie basiert auf der Grundtypologie 'Arbeitsmarktpotenzial' und auf den zwei daraus abgeleiteten Zielgruppendimensionen 'Leistungsfähigkeit' und 'Schulgewohntheit' (siehe BFM 2009b). Der Einfachheit halber wurden nur jeweils zwei Ausprägungen unterschieden:

(1) Leistungsfähigkeit: Leistungsfähig bedeutet, dass die gesundheitlichen Voraussetzungen für die Integration in den ersten Arbeitsmarkt gegeben sind. Leistungsbeeinträchtigt meint, dass gesundheitliche Beeinträchtigungen vorliegen, auf Grund derer eine Integration in den ersten Arbeitsmarkt eher unwahrscheinlich ist.

(2) Schulgewohntheit: Unter schulgewohnt wird verstanden, dass mindestens sechs Jahre Grundschule beziehungsweise weiterführende Schulen und Ausbildungen vorzuweisen sind und der schulisch strukturierte Wissenserwerb der betroffenen Person vertraut ist. Schulungewohnt sind Teilnehmende mit weniger als sechs Jahren Grundschule und / oder mit so lange zurückliegender Schulzeit, sodass wichtige schulische Fähigkeiten verloren gegangen sind.

Aus der Kombination der beiden Zielgruppentypologien lassen sich die vier Haupt-Zielgruppen ableiten:

(1) leistungsbeeinträchtigte, schulungewohnte Personen $\rightarrow$ adäquate Beschäftigung (allenfalls in Kombination mit Qualifikation) als Hauptthema;

(2) leistungsbeeinträchtigte, schulgewohnte Personen $\rightarrow$ adäquate Beschäftigung als Hauptthema;

(3) leistungsfähige, schulungewohnte Personen $\rightarrow$ Qualifikation als Hauptthema oder allenfalls auch Arbeitsmarktintegration;

(4) leistungsfähige, schulgewohnte Personen $\rightarrow$ Arbeitsmarktintegration als Hauptthema, falls nicht möglich adäquate Beschäftigung.

Bei den Zielgruppen 1 bis 4 handelt es sich nicht um trennscharfe Gruppen. Der Sinn der Typologie liegt in einer möglichst einfachen und dennoch sinnvollen $\mathrm{Zu}$ ordnung von Programmteilnehmenden in Bezug auf deren Arbeitsmarktfähigkeit. 
Diese Grundtypologie und die entsprechenden Haupt-Zielgruppen können ergänzt und differenziert werden durch weitere relevante Zielgruppendimensionen wie zum Beispiel Alter, Deutschkenntnisse oder Betreuungspflichten. Die Definition der Zielgruppen legte den Grundstein dazu, die Bildungs-, Beschäftigungs- und Integrationsangebote näher zu definieren und dabei zu beschreiben, welche Zielsetzungen damit verbunden sind. Gleichzeitig wurde die Zulassung der Zielgruppentypen zu jedem Programmtyp festgelegt. Damit war eine konzeptuelle Verknüpfung zwischen Programm- und Zielgruppentypen geschaffen, die eine Analyse und Überprüfung der Angebote ermöglichte (siehe Abschnitt 5.2.2).

Hinweis auf die Umsetzung der Gestaltungsaufgabe 'Angebots- und Zielgruppenanalysen' in den Anwendungsbeispielen

Entwicklung des Versorgungskonzepts «Gesundheit und Alter» der Gemeinde Kriens (Abschnitt 5.3):

Die Trägerinstanz der ambulanten Krankenpflege in Kriens führt seit 2006 eine systematische Übersicht über alle Angebote zu Alter und Gesundheit in der Gemeinde. Dieses laufend aktualisierte Angebotsinventar bildete die Grundlage für die Angebotsanalyse. Eine Zielgruppenanalyse wurde ebenfalls durchgeführt. Sie zeigte, dass die Zahl der älteren Personen (65 Jahre und älter) in der Gemeinde Kriens bis 2030 um rund 60\% ansteigen wird. Die Zahl der pflegebedürftigen Personen wird von 450 Personen (2010) auf rund 800 Personen (2030) wachsen. Parallel dazu wird auch die Zahl der hilfsbedürftigen Personen zunehmen (von ca. 1'000 auf ca. 1'500 Personen).

\subsubsection{Gestaltungsaufgabe A3: Versorgungsstrukturanalyse}

In Ergänzung zu den Angebots- und Zielgruppenanalysen widmet sich die Versorgungsstrukturanalyse der Untersuchung und Bewertung der Strukturen in Versorgungsnetzen und befasst sich mit Fragen der Inanspruchnahme von Angeboten, der Abstimmung innerhalb einer Versorgungskette sowie der räumlichen Gestaltung der Versorgungsnetze. Im Zentrum der Untersuchung stehen folgende Problemkreise:

- $\quad$ Zugang zum Angebot / Erreichbarkeit des Angebots: Werden die anvisierten Zielgruppen mit dem Dienstleistungsangebot überhaupt erreicht?

- $\quad$ Vermittlung / Zuweisung: Erfolgt die Zuweisung zum Angebot strukturiert und nach zweckmässigen Kriterien, sodass eine optimale Passung zwischen dem Zielgruppenprofil und dem Angebotsprofil erreicht werden kann? 
- $\quad$ Angebotsabstimmung in der Versorgungskette: Sind die Angebote der Einrichtungen in der Versorgungskette zweckmässig aufeinander abgestimmt (keine Doppelspurigkeiten oder Lücken) und hinreichend durchlässig? Wie gut funktioniert die Zusammenarbeit zwischen den Einrichtungen?

- $\quad$ Räumliche Gestaltung: Sind die Grösse des Einzugsgebiets, die Lage der leistungserbringenden Organisationen im Raum (Erreichbarkeit), deren Spezialisierungsgrad und deren Grösse zweckmässig gestaltet?

Um die formulierten Herausforderungen in Versorgungsstrukturen $\mathrm{zu}$ untersuchen, erweisen sich Befragungen von involvierten Fachpersonen sowie von Nutzerinnen und Nutzern als hilfreich. Da es in der Regel noch nicht hinreichend bekannte Zusammenhänge in Versorgungsstrukturen auszuloten gilt, sind strukturierte Leitfadeninterviews empfehlenswert. Für die Analyse des Angebotszugangs und die Untersuchung von Vermittlungs- und Zuweisungsfragen ist die Befragung von Nutzerinnen und Nutzern notwendig.

Voraussetzung für eine Analyse der Angebotsabstimmung ist die Beschreibung und Definition entsprechender Versorgungsketten. Eine Identifizierung von Versorgungsketten ist beispielsweise entlang der Unterscheidung von Primäroder Basisversorgung, erweiterter Versorgung und (hoch) spezialisierter Angebote möglich.

Beispiel - Analyse der Angebotsstrukturen für ältere Menschen in einer mittelgrossen Gemeinde

Aus einer Analyse der Angebotsstrukturen einer mittelgrossen Gemeinde durch ein Team der Hochschule Luzern (von Bergen/Bieri 2010) resultierten im Bereich der Versorgungsstrukturen für ältere Menschen folgende Ergebnisse:

- $\quad$ Räumliche Lage: Die befragten Fachpersonen wiesen auf den Standort des Alters- und Pflegeheims ausserhalb des Zentrums hin. Grundsätzlich sollten Wohn- und Hilfsangebote zentral domiziliert sein. Zudem machten die Befragten darauf aufmerksam, dass die Erschliessung des Heims mit öffentlichen Verkehrsmitteln zu optimieren wäre.

- Wenig institutionalisierte Zusammenarbeit: Verschiedene Fachpersonen konstatierten, dass die Zusammenarbeit zwischen den zahlreichen Einrichtungen offensichtlich vor allem aufgrund persönlicher und individueller Kontakte verlaufe und weitgehend auf freiwilliger Basis funktioniere. Verschiedentlich wurde auf ungenützte Synergien zwischen den Institutionen aufmerksam gemacht. Einige der Interviewpartner/-innen vermissten institu- 
tionalisierte Formen der Zusammenarbeit wie etwa regelmässige «Austauschgefässe» für alle Akteure im Altersbereich oder gar eine gemeinsame und koordinierte Führung der wichtigsten Angebote der Altershilfe. Zusammenarbeitspotenziale in der Region würden zudem zu wenig genutzt.

Hinweise auf die Umsetzung der Gestaltungsaufgabe 'Versorgungsstrukturanalyse' in den Anwendungsbeispielen

Überprüfung der BBIP im Kanton Zürich (Abschnitt 5.2):

Die Analyse der Angebotskette (Abschnitt 5.2.2) zeigte, dass bei der Zuweisung der Flüchtlinge und vorläufig Aufgenommenen ein Kernproblem liegt: Wie kommen die Klientinnen und Klienten in das für sie geeignete Programm? Mit einer Konzipierung und Einführung einer Triage-Assessment-Stelle sollten die Information, Koordination und Steuerung beim BBIP-Angebot verbessert werden beziehungsweise eine sinnvolle Zuweisung in einzelne Programme sowie eine längerfristige Prozessbegleitung der Teilnehmenden gewährleistet werden.

Entwicklung des Versorgungskonzepts «Gesundheit und Alter» der Gemeinde Kriens (Abschnitt 5.3):

In Kriens wurde die Analyse der Versorgungsstrukturen mit einer besonderen Vertiefung in Bezug auf die Wechselwirkungen zwischen stationärer und ambulanter Versorgung durchgeführt (Abschnitt 5.3.2). Die Strukturanalyse zeigte, dass die Zuweisung zu den Angeboten individuell, punktuell und unkoordiniert erfolgte und keine Zuweisung bestand, die sich an Kriterien orientierte. Zudem fehlten eine systematische Abstimmung der Angebote und eine institutionalisierte Zusammenarbeit mehrheitlich. Die Abklärungen zeigten im Weiteren, dass eine Koordination der Angebote sowie ein fachlicher Austausch von allen Beteiligten gewünscht wurden. 


\subsubsection{Gestaltungsaufgabe A4: Analyse der Leistungserbringenden}

Im Rahmen einer Situationsanalyse werden auch die Merkmale der dem Versorgungssystem zugehörigen Einrichtungen analysiert (z.B. Einrichtungsgrösse, Angebotspalette). Die Einrichtungsanalyse dient der Bewertung der Strukturen, Prozesse und Ergebnisse der Einrichtungen innerhalb eines Versorgungssystems und fokussiert folgende Fragen:

- $\quad$ Sind die Prozesse und Strukturen der jeweiligen Einrichtung organisatorisch effizient gestaltet?

- $\quad$ Sind die organisatorischen Rahmenbedingungen (z.B. Personalressourcen, Qualifikationen des Personals) angemessen ausgestaltet?

- Werden die Qualitätsvorgaben eingehalten?

- Werden die Dienstleistungen effektiv und effizient erbracht?

\section{Beispiel - Verfahren zur Qualitätsüberprüfung}

Als Beispiele für Einrichtungsanalysen können «klassische» Verfahren zur Qualitätsüberprüfung von Einrichtungen (z.B. Einschätzung der Struktur-, Prozess- und Ergebnisqualität) aufgeführt werden. In diesem Bereich stehen verschiedene Überprüfungsinstrumente und Qualitätsmanagementsysteme zur Verfügung; auf sie wird an dieser Stelle nicht ausführlicher eingegangen. In letzter Zeit gewinnt zudem der direkte Vergleich von Einrichtungen anhand von definierten Leistungsund Strukturindikatoren an Bedeutung. In diesem Zusammenhang wird von sogenannten Kennzahlenvergleichen oder Benchmarkings gesprochen. Wie im Fall der schweizerischen «Städteinitiative Sozialpolitik» (Beyeler/Salzgeber/Schuwey 2015) schliessen sich verschiedene Einrichtungen zusammen und lassen ihre Performance in ausgewählten Bereichen - zum Beispiel für die Sozialhilfe - durch ein Set von Kennzahlen in regelmässigen Abständen vergleichen.

Hinweis auf die Umsetzung der Gestaltungsaufgabe 'Analyse der Leistungserbringenden' in den Anwendungsbeispielen

Überprüfung der BBIP im Kanton Zürich (Abschnitt 5.2):

Die sogenannte BBIP-Konzeption 2010 (Bürgisser/Mey 2011) definierte zentrale Eckwerte und Vorgaben seitens der kantonalen Verantwortungstragenden zur Gestaltung des Angebots. Dabei ging es einerseits darum, die Zielgruppen und die Zulassung in die jeweiligen Programme zu bestimmen sowie die vier 
Angebotstypen mit den darauf abgestimmten Zielsetzungen zu definieren. Darauf aufbauend stellte sich andererseits die Frage des Qualitätsmanagements. Bisher hatten die einzelnen Programmanbietenden kein identisches Verfahren, um die Qualität auszuweisen. Für das KSA war zentral, in Bezug auf die Qualität der Angebote einheitliche Standards zu setzen. Gleichzeitig wurde darauf geachtet, das Qualitätsmanagement nicht nur als Legitimationsnachweis zu sehen, sondern es auch gleichzeitig zur fachlichen Weiterentwicklung der Programme zu verwenden. Deshalb sollte kein zu grosser bürokratischer Aufwand betrieben werden, sondern das Qualitätsmanagement sollte Anlass sein, um für die professionelle Arbeit gewisse Standards zu setzen. In diesem Sinn wurden die Qualitätskriterien massgeschneidert für die BBIP-Anbietenden entwickelt.

\subsection{Gestaltungsphase 2: Strategie (S)}

Der zweite Schritt im Gestaltungskreislauf ist die Formulierung einer auf der Situationsanalyse basierenden Strategie im Sinn von konkretisierten Zielsetzungen. Dabei sind auf den vier Gestaltungsebenen je unterschiedliche Inhalte im Fokus, die alle auf die Zielsetzung des Gestaltungsprozesses eines Versorgungssystems ausgerichtet werden müssen:

(S1) die Formulierung von übergeordneten Zielen und Vorgaben (Ebene 1: 'Programm und Finanzierung');

(S2) die Planung von Angeboten und Leistungen (Ebene 2: 'Leistungen und Angebote');

(S3) die Formulierung von finanziellen, prozeduralen und fachlichen Rahmenvorgaben (Ebene 3: 'Versorgungsstrukturen');

(S4) die Konzeption von Leistungsvereinbarungen (Ebene 4: 'Leistungserbringung').

Die folgende Abbildung gibt einen Überblick über die Aufgaben in der Phase der Strategieentwicklung. 


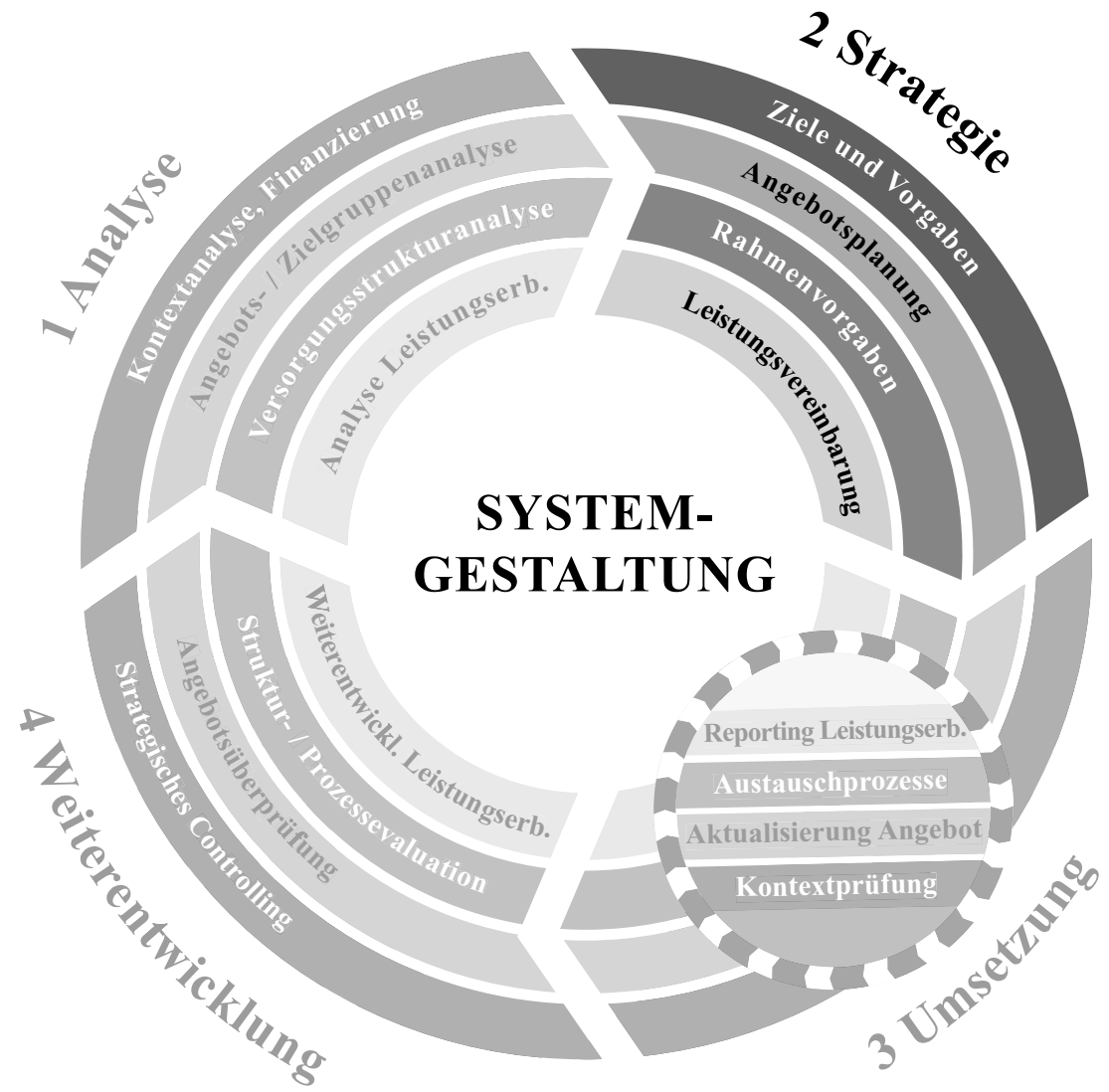

Abbildung 18: Strategieentwicklung in der Systemgestaltung: Phase 2 Quelle: eigene Darstellung

\subsubsection{Gestaltungsaufgabe S1: Ziele und Vorgaben}

Die Basis für die Definition von Zielen sind in der Regel sozialpolitische Entwicklungen, die Handlungsdruck erzeugen. Im Rahmen von politischen Diskursen und Prozessen kann dies zu veränderten Vorgaben und Rahmenbedingungen wie zum Beispiel Veränderungen in der Gesetzgebung führen, die dann durch die Verwaltung umgesetzt werden. Bei der Systemgestaltung durch staatliche Stellen (Bund, 
Kantone, Gemeinden) bilden oft neue oder angepasste Gesetze und Verordnungen die übergeordneten Ziele ab. Zudem können auch bestehende gesetzliche Grundlagen aufgrund von sozialpolitischem Handlungsdruck anders ausgelegt werden und zu veränderten strategischen Zielen führen. Auch private Einrichtungen wie zum Beispiel national tätige soziale NPO reagieren auf sozialpolitische Entwicklungen und können aufgrund ihrer Zweckbestimmung eigene, übergeordnete Ziele setzen.

Zur Entwicklung von Strategieprozessen für Unternehmen und Organisation besteht eine reichhaltige Literatur. Henry Mintzberg, Bruce Ahlstrand und Joseph Lampel (2012) beschreiben in ihrem Klassiker «Strategy Safari» zehn unterschiedliche Denkschulen, wovon die Gestaltungsschule, welche die Strategiebildung als konzeptionellen Prozess versteht, in der Managementlehre und der Praxis weit verbreitet ist. Als Kernelemente werden bei dieser Schule einerseits die Umfeldentwicklungen im Sinn einer äusseren Beurteilung von relevanten Faktoren und anderseits die Organisation mit ihren Stärken und Schwächen analysiert. Der Strategieprozess im Rahmen der Systemgestaltung hat eigene Gesetzmässigkeiten; es lassen sich aber einige Analogien zur Gestaltungsschule nach Mintzberg, Ahlstrand und Lampel (2012: 45) herstellen. Anstelle einer Umfeldanalyse aus der Perspektive einer einzelnen Organisation können der - für ein bestimmtes Versorgungsystem relevante - Diskurs sozialer Probleme und der daraus resultierende sozialpolitische Handlungsdruck auf Politik und Gesetzgebung herangezogen werden. Dieser Prozess entspricht der vorher beschriebenen Analysephase (A1), 'Kontextanalyse, Finanzierung und Realisierbarkeit'. Auf Basis dieser Kontextanalyse können langfristige, übergeordnete Ziele und Vorgaben für ein bestimmtes soziales Programm und das entsprechende Versorgungssystem festgelegt werden. Analog zur Organisationsanalyse gemäss Gestaltungsschule können die Analyseschritte 'Angebots- und Zielgruppenanalyse' (A2), 'Versorgungsstrukturanalyse' (A3) sowie 'Analyse der Leistungserbringenden' (A4) aufzeigen, ob ein Versorgungsystem den übergeordneten Zielen entsprechend aufgestellt ist oder ob bestimmte Anpassungen geplant werden müssen.

\section{Beispiel - Neuausrichtung der Alterspolitik in Schaffhausen}

In der Stadt Schaffhausen leben überdurchschnittlich viele ältere Menschen. Auf kantonaler Ebene liegen diesbezüglich nur die Kantone Basel-Stadt und Tessin vor dem Kanton Schaffhausen. Auf dem Hintergrund dieses Sachverhalts wurde bereits 2003 - unter dem Einbezug breiter Kreise - eine öffentliche Diskussion zur zukünftigen Alterspolitik geführt. Im Jahr 2008 beschloss die Stadt Schaffhausen eine Neuausrichtung ihrer Alterspolitik. Mittels vielfältiger Massnahmen wurde 
und wird «zuhause alt werden» als eine Option für möglichst viele ältere Menschen in Schaffhausen angestrebt. Dazu wurden drei Handlungsansätze verfolgt, nämlich erstens die Förderung altersgerechter vielfältiger Wohnformen, zweitens die Bereitstellung einer breiten Palette pflegerischer und hauswirtschaftlicher Dienstleistungen, die niederschwellig zugänglich sind, den persönlichen Bedarf berücksichtigen und möglichst wohnortsnah zu beziehen sind, und drittens die Förderung altersfreundlicher Quartierstrukturen und der sozialen Einbindung von älteren Menschen (Stocker 2015). Die Angebote für ältere Menschen sollten dezentral in Quartierdienstleistungszentren (QDZ) gebündelt werden. Diese sollen unterschiedliche stationäre Wohn- und Pflegeangebote sowie eine Vielzahl von ambulanten Dienstleistungen besonders quartiernah anbieten, insbesondere die Integration eines ambulanten Pflegeteams vor Ort. Zu den Zielen der QDZ gehörte auch die soziale Vernetzung der Senior/-innen im jeweiligen Quartier.

Das Beispiel macht deutlich sichtbar, wie aufgrund von gesellschaftlichen Entwicklungen (Demografie) Handlungsdruck aufgebaut und nach vorgängigen Analysen eine übergeordnete Zielsetzung und Strategie in Form eines Beschlusses der Legislative (Grosser Stadtrat) in Kraft gesetzt wurde. Das Beispiel zeigt auch, dass solche übergeordneten Zielsetzungen langfristig ausgerichtet sind und die Umsetzung mehrere Jahre in Anspruch nehmen kann, wobei die Ausrichtung der Strategien auch immer wieder hinterfragt und überprüft werden muss.

Hinweise auf die Umsetzung der Gestaltungsaufgabe 'Übergeordnete Ziele und Vorgaben' in den Anwendungsbeispielen

Übergabe der Behindertenhilfe vom Bund an die Kantone am Beispiel des Kantons Zug (Abschnitt 5.1):

Eine wichtige Grundsatzentscheidung, die bei der Umsetzung der Neuordnung der Finanzierung und Aufgabenteilung zwischen Bund und Kantonen im Bereich der Behindertenhilfe im Kanton Zug (siehe Abschnitt 5.1.2) zu treffen war, war die Wahl der zukünftigen Form der Finanzierung. Sollte von einer Objektzu einer Subjektfinanzierung gewechselt werden, also weg von der Finanzierung der Einrichtungen hin zu einer direkten Finanzierung der Menschen mit Behinderung? Nach sorgfältiger Prüfung und Abwägung entschieden sich die Verantwortlichen, grundsätzlich am Modell der Objektfinanzierung festzuhalten. Diese Vorgabe wirkte sich auf die weitere Ausgestaltung des Prozesses und die weiteren Phasen des LUS bis hin zu den Leistungsvereinbarungen mit den Einrichtungen aus. 
Überprüfung der BBIP im Kanton Zürich (Abschnitt 5.2):

Ein wichtiger Meilenstein im Entwicklungsprozess der BBIP war die Verabschiedung der BBIP-Konzeption von 2010 (Bürgisser/Mey 2011), die einheitliche konzeptuelle Grundlagen und Anforderungen an das Qualitätsmanagement der BBIP formuliert. Parallel dazu wurde das Controlling verbessert und auf das dazugehörige Qualitätsmanagement abgestimmt. Der letzte Schritt zur Weiterentwicklung des Controllings lag in der Einführung von Finanzkennzahlen im Hinblick auf eine bessere Vergleichbarkeit der Kosten.

\subsubsection{Gestaltungsaufgabe S2: Angebotsplanung}

Angebotsplanungen basieren auf den übergeordneten strategischen Zielen und Rahmenvorgaben sowie auf der Grundlage von Bedarfsanalysen und Bedarfsprognosen. Im Rahmen der Angebotsplanung wird festgelegt, welche Angebote und Leistungen erbracht werden sollen und wer für die Leistungserbringung zuständig ist. Bei der öffentlichen Verwaltung wird die Angebotsplanung häufig in Form von Planungsberichten abgebildet, die anschliessend von den zuständigen politischen Instanzen genehmigt werden. Bei privaten Organisationen wird die Angebotsplanung oft im Rahmen der Operationalisierung eines Strategiepapiers festgehalten.

Beispiel - Neugestaltung der Angebotsplanung in der Altenhilfe der Stadt Schaffhausen

Die Angebotsplanung in der Altenhilfe der Stadt Schaffhausen ${ }^{13}$ richtete sich ab 2008 auf die übergeordnete Zielsetzung «zuhause alt werden» aus. Obengenannte QDZ sollten als Herzstück eines Versorgungssystems mit stationären und ambulanten Dienstleistungen aufgebaut werden. Die bisher bestehenden Alters- und Pflegeheime sollten ihr Angebot auf diese neue Strategie ausrichten. Auch für die bis 2008 zentral geführte ambulante Pflege (Spitex) hatte diese Angebotsplanung weitreichende Konsequenzen, da die zentrale Struktur aufgelöst und die Dienstleistungen der Spitex den QDZ zugeordnet werden sollten. Eine derart weitreichende Neuausrichtung konnte nur über einen längeren Zeitrahmen mit einem schrittweisen Vorgehen angestrebt werden. Als Pilotprojekt wurde im Herbst 2010 das QDZ Künzle-Heim eröffnet und ein Spitex-Team wurde dort vor Ort inte- 
griert. Mit diesem Schritt wurden unterschiedliche Wohn- und stationäre Pflegeangebote mit einer Palette von ambulanten Dienstleistungen kombiniert und auf die neue übergeordnete Zielsetzung ausgerichtet. Gemäss Planung sollten 2015 drei weitere QDZ folgen. 2012 wurde die bisher privat geführte Spitex zu einem städtischen Angebot, damit die Dezentralisierung umgesetzt werden konnte. Als begleitende Massnahmen wurden Ambulatorien in den Spitex-Räumlichkeiten der QDZ eingerichtet. Sie sind für die Quartierbevölkerung zu bestimmen Öffnungszeiten zugänglich. Kleine medizinische Versorgungen sowie Beratungen zu gesundheitlichen Themen für Betagte und für pflegende Angehörige werden angeboten. Die Abklärung von möglichen Heimeintritten wurde einer zentralen Beratungsstelle im Bereich Betreuung der Stadt Schaffhausen zugeordnet. Weiter wurde eine Fachstelle für Gemeinwesen orientierte Gesundheitsförderung geschaffen. Um altersfreundliche Quartierstrukturen zu erhalten oder zu schaffen und die älteren Menschen sozial einzubinden, wurde eine Stabstelle Quartierentwicklung aufgebaut.

Hinweis auf die Umsetzung der Gestaltungsaufgabe 'Angebotsplanung' in den Anwendungsbeispielen

Entwicklung des Versorgungskonzepts «Gesundheit und Alter» der Gemeinde Kriens (Abschnitt 5.3):

Aus dem Versorgungskonzept (siehe Abschnitt 5.3.3) geht hervor, dass auf der Basis der strategischen Zielsetzung eine detaillierte Angebotsplanung mit den folgenden Elementen festgelegt wurde:

- $\quad$ Optimierung und Vernetzung des bestehenden Angebots durch eine Koordinations- und Anlaufstelle als «Drehscheibe» (Information und Beratung sowie Koordination und Weiterentwicklung);

- $\quad$ Förderung von betreuten Wohnformen;

- $\quad$ Pflegeplätze in Pflegewohnungen (zwei Wohnungen bis 2020, später allenfalls mehr, je nach Bedarf) im Sinn eines flexiblen Systems;

- $\quad$ Optimierung des bestehenden Angebots an Heimplätzen (steigender Pflegebedarf);

- $\quad$ Ausbau der Entlastungsangebote zur Unterstützung der pflegenden Angehörigen;

- $\quad$ Stärkung der Freiwilligenarbeit (Freiwilligenagentur);

- $\quad$ regionaler Ansatz: Kooperationen mit Nachbargemeinden. 


\subsubsection{Gestaltungsaufgabe S3: Rahmenvorgaben}

Übergeordnete Zielsetzungen und in Planungsberichten beschriebene Leistungen und Angebote müssen weiter konkretisiert werden. Dazu werden definierte finanzielle, prozedurale und fachliche Rahmenvorgaben benötigt. Bei diesem Schritt sind die finanziellen Möglichkeiten und Grenzen wegleitend. Der finanzielle Spielraum muss bekannt sein, damit die Erbringung von Leistungen, die Konzeption von Angeboten, Qualitätsstandards sowie Abläufe und Prozesse näher definiert werden können.

Es wird festgelegt, wie die Versorgung konkret gestaltet werden soll: Welche Leistungen werden angeboten? Wer erbringt die Leistungen? Welche Qualitätsstandards gelten? In welcher Form soll das Versorgungssystem gestaltet werden? Erbringt die öffentliche Hand die Leistungen oder besteht eine Zusammenarbeit mit privaten Einrichtungen? Gibt es Ausschreibungen beziehungsweise Wettbewerbe oder werden die Aufträge direkt vergeben? Bei national tätigen privaten Organisationen stellt sich die Frage, wie die Leistungen operationalisiert werden können und sollen: Sind zentrale oder dezentrale Strukturen vorhanden und zu nutzen?

Auch bei der Festlegung der Rahmenvorgaben für Versorgungssysteme sind die Ergebnisse der Situationsanalyse zu berücksichtigen; deren Teilaufgaben wurden im Abschnitt 4.2 erörtert. Im Grundmodell der Gestaltungsschule nach Mintzberg, Ahlstrand und Lampel (2012: 45) entspricht die Analyse bereits bestehender Angebote, Leistungen und Rahmenvorgaben (Strategiebildung als konzeptioneller Prozess) der «inneren Beurteilung». Während beim Strategieprozess einer einzelnen Organisation eine 'Organisationsanalyse' vorausgesetzt wird, stellen bei Strategieprozessen im Rahmen der Systemgestaltung die Angebotsüberprüfung und der Check der Versorgungsstrukturen die vergleichbare zentrale Voraussetzung dar.

\section{Beispiel - Rahmenvorgaben zur Neuausrichtung der Alterspolitik in der Stadt Schaffhausen}

Die Operationalisierung der Zielsetzung, ein umfassendes dezentrales Wohn- und Pflegeangebot aufzubauen und zu implementieren, erforderte von den Verantwortlichen in Schaffhausen eine detaillierte Konzeptarbeit. Es wurde eine eigene Projektorganisation eingesetzt mit der Vorgabe, Strukturen im Bereich der Betreuung so anzulegen, dass sie dem Bedarf durch die demografischen und gesellschaftlichen Entwicklungen entsprechen konnten und können. Im Rahmen dieser umfassenden Neuorganisation des gesamten Versorgungssystems für die ältere Bevölkerung waren Rahmenvorgaben im Sinn einer verbindenden Klammer wichtig, 
damit das Projekt als Ganzes kohärent umgesetzt werden konnte. So wurde beispielsweise eine gemeinsame Philosophie der unterschiedlichen Akteure zur Altersbetreuung entwickelt und schrittweise etabliert. Das Dienstleistungsangebot im stationären und ambulanten Bereich musste den zentralen und dezentralen Akteuren klar und verbindlich zugewiesen werden. Dabei musste sichergestellt werden, dass die Dienstleistungen bei Bedarf weiter ausgebaut und flexibel angepasst werden konnten und können. Administrative Aufgaben und Prozesse mussten auf das neue Gesamtkonzept ausgerichtet und mit entsprechenden Ressourcen ausgestattet werden. Die Strategieentwicklung und die Führung des Entwicklungsprojekts und des daraus entstandenen Bereichs der Betreuung innerhalb der Stadtverwaltung wurden durch eine neu gebildete Geschäftsleitung wahrgenommen. In der Geschäftsleitung waren neben dem verantwortlichen Stadtrat die Leitung des Bereichs der Betreuung, die Leitungen der QDZ sowie die Stabsstelle Quartierentwicklung Schaffhausen vertreten. Die Neukonzeption sollte ressourcenneutral umgesetzt werden, was zu entsprechenden finanziellen Rahmenvorgaben führte.

Hinweis auf die Umsetzung der Gestaltungsaufgabe 'Rahmenvorgaben für das Versorgungssystem' in den Anwendungsbeispielen

Überprüfung der BBIP im Kanton Zürich (Abschnitt 5.2):

Die Verantwortlichen des Kantons Zürich haben als Rahmenvorgabe eine Zielgruppen- und Programmtypologie festgelegt, an der sich die Programmanbietenden auszurichten haben. In der Zielgruppentypologie sind vier Hauptzielgruppen und in der Programmtypologie ebenfalls vier unterschiedliche Programmtypen definiert worden. Jede anbietende Stelle hat sich auf einen der vier Programmtypen festzulegen und zu definieren, auf welche Zielgruppen sich das Programm bezieht. Unter Bezugnahme auf die zielgruppenspezifischen Bedürfnisse und den entsprechenden Programmtyp definiert die anbietende Stelle die Ziele ihres Angebots. Schliesslich werden die inhaltliche und zeitliche Programmgestaltung sowie die Prozessqualität zur Erreichung der formulierten Ziele entwickelt und festgelegt.

\subsubsection{Gestaltungsaufgabe S4: Leistungsvereinbarung}

Die übergeordneten Ziele, die fachlichen und finanziellen Vorgaben sowie die Eckwerte der Angebotsplanung bilden die Grundlage für die Ausarbeitung der Leistungsvereinbarungen mit den einzelnen Einrichtungen. Form, Art und Umfang der Vereinbarungen können dabei unterschiedlich ausgestaltet sein. Bei der 
Vergabe von öffentlichen Aufgaben an private Einrichtungen hat sich das Leistungsauftragsmodell weitgehend durchgesetzt, wobei in der Praxis grosse Unterschiede bezüglich Detaillierungsgrad und Finanzierungsmodus (z.B. Objekt- oder Subjektfinanzierung) anzutreffen sind. In der Regel bestehen auch für Einrichtungen der öffentlichen Hand verwaltungsinterne Leistungsaufträge.

Bei der Formulierung von Leistungsaufträgen ist die leistungsbeziehende Zielgruppe zentral. Sinn und Zweck der sozialen Dienstleistung, die in der Regel die Lebenslage von bestimmten Bevölkerungsgruppen verbessern soll, dürfen nicht organisationalen Sachzwängen zum Opfer fallen.

\section{Beispiel - Leistungsvereinbarung mit einem QDZ in Schaffhausen}

Bei diesem Umsetzungsschritt der Systemgestaltung handelt es sich um die Leistungen, die von einzelnen Akteuren eines Versorgungssystems erbracht werden sollen. Am Beispiel des QDZ Künzle-Heim, das als Pilotprojekt geführt wurde, kann dies konkretisiert werden. Der Leistungskatalog für 2014 wurde im Bericht des ETH-Wohnforums wie folgt umschrieben (Hugentobler/Wurster 2013):

Wohn-, Pflege- und Betreuungsangebote für rund 120 Bewohner/-innen:

- $\quad$ selbstständiges Wohnen: 30 1- und 2-Zimmer-Wohnungen;

- betreutes Wohnen: 57 Einzelzimmer mit Nasszellen;

- Demenzabteilung: Wohngruppe mit acht Zimmern;

- halbambulantes Angebot: drei Zimmer als Ferienplätze, für Tages- oder Nachtaufenthalt.

Durch QDZ angebotene Dienstleistungen:

- $\quad$ Spitex-QDZ Künzle-Heim;

- Ambulatorium;

- Beratung «Gesundheitsförderung \& Prävention»;

- Mittagstisch;

- $\quad$ hauswirtschaftliche Dienstleistungen (Reinigungsdienst und Wäscheservice);

- Altersgymnastik, Bewegungs- und Gehtraining, Kraftraum, Infrarotkabinen, Wassermassageliege;

- «Treffpunkt QDZ», wöchentlich für Interessierte aus dem Quartier:

- Angebote und Anlässe zur Freizeitgestaltung und Unterhaltung. 
Angebote von Dritten im QDZ Künzle-Heim:

- Physiotherapie;

- Friseur;

- Podologie;

- Mütter- und Väterberatung, Kanton Schaffhausen;

- $\quad$ Sozialberatung der Pro Senectute, monatlich.

Jede einzelne dieser Leistungen muss genauer mit Eckwerten zu qualitativen und quantitativen Elementen und zur Ressourcenausstattung festgelegt, umschrieben und erfasst werden. Nur so wird eine betriebswirtschaftliche Steuerung möglich. Gemäss Regula Ruflin und Andreas Dvorak (2007: 75ff.) muss ein Leistungsvertrag mindestens über die folgenden Gegenstände Auskunft geben:

- Vertragsparteien;

- $\quad$ gesetzliche Grundlagen und andere dem Kontrakt zugrundeliegende Reglemente, Konzepte oder Richtlinien;

- Zweck: Übergeordnete Ziele / Strategie (bezogen auf die Politik, das Sachgebiet und die Anspruchsgruppen, auf welche sich die im Leistungsauftrag geregelte Leistungserbringung bezieht);

- Leistungen / Produkte: Art der Leistungen / Produkte, Inhalt der Leistungen / Produkte, Quantität / Anzahl der Leistungen / Produkte, quantitative und qualitative Qualitäts- respektive Wirkungskriterien dieser Leistungen / Produkte;

- allgemeine und spezifische Beschreibung der Zielgruppen der Leistungen / Produkte;

- finanzielle Abgeltung: Tarifberechnungsmodalitäten (inkl. Faktoren wie Regelung von Teuerungszulagen oder Übernahme gewisser Steuern), Gesamtbetrag, Abrechnungs- und Auszahlungsmodalitäten, Umgang mit Überschüssen und Verlusten, finanzielles Controlling: Revisionsvorgaben und Kennzahlen;

- Modalitäten des Berichtswesens: Monitoring und Reporting;

- Gültigkeits- / Geltungsbereich des Leistungsvertrags: Dauer, Vertragsbeginn und Vertragsende, geografische Reichweite;

- Änderungs-, Rücktritts- und Auflösungsmodalitäten;

- Verlängerungs- / Neuverhandlungsmodalitäten;

- $\quad$ Folgen bei Nicht- oder ungenügender Erfüllung des Vertrags (Schlecht- oder Nichterfüllung durch die auftragnehmende respektive durch die auftraggebende Organisation);

- Rechtsmittelbelehrung und Gerichtsstand;

- Ort, Datum und Unterschrift der Vertragsparteien. 


\subsection{Gestaltungsphase 3: Umsetzung (U)}

Der Gestaltungskreislauf in Versorgungssystemen zeichnet sich im Wesentlichen durch einen mehrjährigen Rhythmus aus. Umfassende Angebotsanalysen, Zielbestimmungen oder vertiefte Überprüfungs- und Weiterentwicklungsanstrengungen werden in der Regel alle paar Jahre durchgeführt. Die laufende Umsetzung dieser gehört indes zum alltäglichen Handeln der involvierten Akteure und daher folgt dieser dritte Schritt einem anderen zeitlichen Rhythmus.

Die Umsetzung und Operationalisierung der Vorgaben und Ziele ist eine Aufgabe, die permanent wahrgenommen wird. Sie umfasst entlang der vier Gestaltungsebenen folgende Aufgaben:

(U1) reflexive Kontext- und Systemüberprüfung (Ebene 1: 'Programm und Finanzierung');

(U2) laufende Aktualisierung des Angebots (Ebene 2: 'Leistungen und Angebote');

(U3) Austauschprozesse im Versorgungssystem (Ebene 3: 'Versorgungsstrukturen');

(U4) Reporting der leistungserbringenden Einrichtungen (Ebene 4: 'Leistungserbringung').

Die folgende Abbildung gibt einen Überblick über die Aufgaben in der Phase der Umsetzung. 


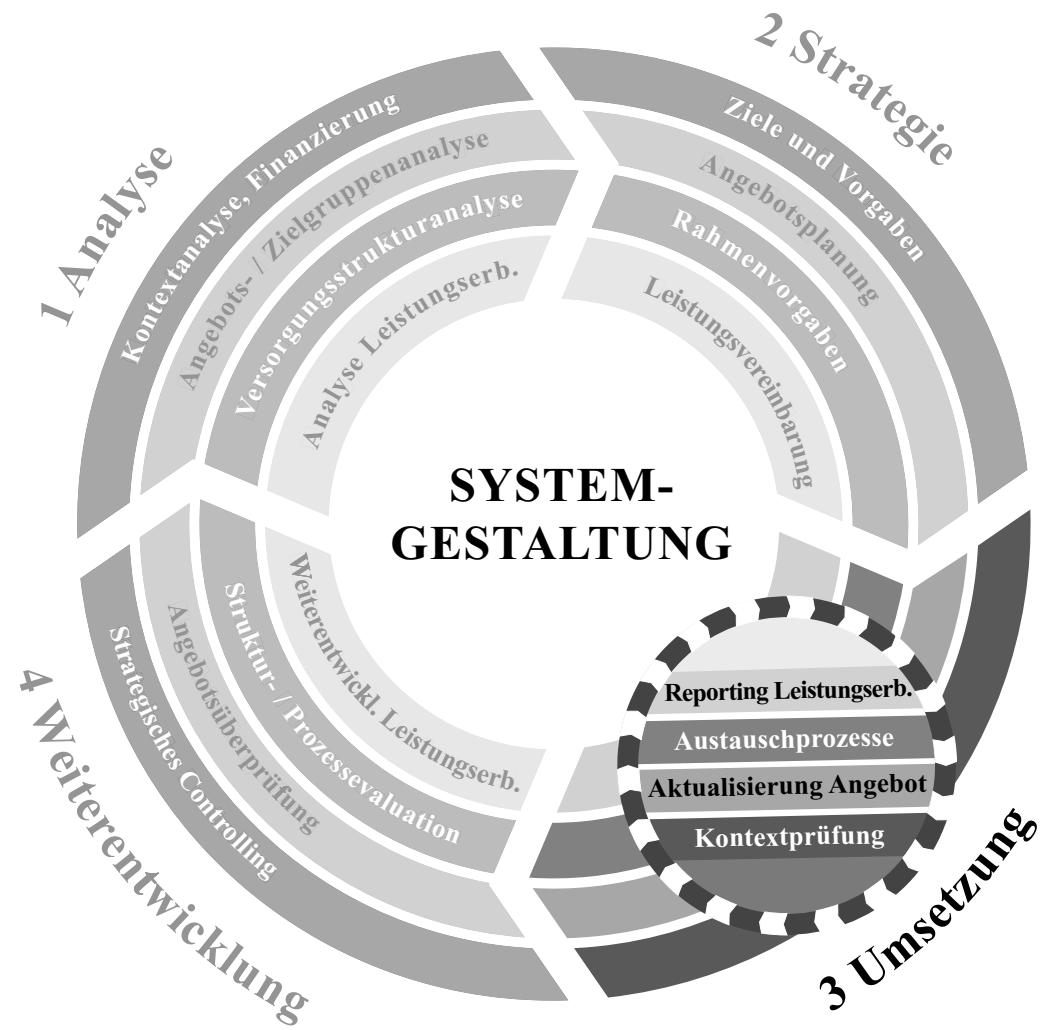

Abbildung 19: Umsetzung in der Systemgestaltung: Phase 3 Quelle: eigene Darstellung

\subsubsection{Gestaltungsaufgabe U1: Kontextprüfung}

Die Leistungserbringung ist laufend neuen gesellschaftlichen Trends und Entwicklungen (Umwelt) ausgesetzt. Diese gilt es ständig zu beobachten und - sofern möglich - in der laufenden Planung und Steuerung zu berücksichtigen. Das Gleiche gilt für Veränderungen wichtiger gesetzlicher, finanzieller oder struktureller Vorgaben und Rahmenbedingungen. Gefragt ist ein eigentliches Monitoring im Sinn einer aufmerksamen, ständigen Beobachtung der relevanten Entwicklungen, 
damit es möglich ist, bei Bedarf einzugreifen. In der Regel verlaufen solche Änderungen im Sinn eines Wandels erster Ordnung (Vahs 2009). Vahs weist darauf hin, dass es sich dabei um graduelle, evolutionäre Entwicklungen handelt und es in der Regel genügt, die Organisation laufend schrittweise anzupassen ohne grundlegende Veränderung des Bezugsrahmens. Selten, aber nicht auszuschliessen ist auch ein Wandel zweiter Ordnung. Vahs (2009) beschreibt diesen als radikalen, revolutionären, transformativen Wandel, der zu einschneidenden Veränderungen der Organisation und der Arbeitsweise führt.

Beispiel - markante Nachfrageschwankungen bei der Unterbringung und Betreuung von Asylsuchenden und Flüchtlingen in der Schweiz

Bei der Bewältigung der Herausforderungen, die sich bei der Unterbringung und Betreuung von Asylsuchenden und Flüchtlingen stellen, wird der Anspruch nach einem ständigen Monitoring und damit verbunden die Fähigkeit, rasch und flexibel zu reagieren, besonders deutlich. Trotz der vielfältigen gesetzgeberischen Anpassungen auf europäischer und nationaler Ebene ist die Zahl der einreisenden Personen letztlich nicht steuerbar. Bei Bund und Kantonen sowie den beteiligten privaten Organisationen ist daher eine hohe Flexibilität gefragt. Je nach internationaler Lage kann normalerweise von einem sogenannten Regelbetrieb ausgegangen werden. Die Unterbringungs- und Betreuungskapazitäten werden auf eine bestimmte Bandbreite ausgelegt. Das Szenario einer unerwartet raschen Zunahme der Immigration muss aber ständig mitbedacht werden. Das Versorgungssystem muss in der Lage sein, ohne langen Vorlauf mehr Leistung zu erbringen. Die Entwicklungen der Flüchtlingszahlen werden darum vom Staatssekretariat für Migration in einem ständigen Monitoring beobachtet und es besteht ein enger, ständiger Austausch mit den Kantonen und den operativ tätigen Einrichtungen (z.B. Empfangsstellen).

Hinweis auf die Umsetzung der Gestaltungsaufgabe 'reflexive Kontext- und Systemüberprüfung' in den Anwendungsbeispielen

Übergabe der Behindertenhilfe vom Bund an die Kantone am Beispiel des Kantons Zug (Abschnitt 5.1):

Die Leistungserbringung ist nicht Sache des Kantons, sondern Aufgabe der einzelnen privaten Einrichtungen. Aus der Sicht der sozialpolitischen Steuerung ist die Leistungserbringung aber durch die Kantone begleitend zu beobachten und wenn nötig zu koordinieren. Gesellschaftliche, fachliche, politische und / oder finanzielle Entwicklungen, die Einfluss auf die zukünftige Ausrichtung der Versorgungssteuerung haben, werden festgestellt und weiterverfolgt. 


\subsubsection{Gestaltungsaufgabe U2: Aktualisierung des Angebots}

Im Rahmen eines laufenden Abgleichs oder einer Angebotsabstimmung werden die Kapazitäten und (freien) Angebote der Einrichtungen mit den nachgefragten Dienstleistungen abgeglichen. Diese Abgleichungsprozesse können der Eigenregie des «Marktes» der beteiligten Einrichtungen überlassen werden oder direktiver im Rahmen eines Nutzungsmanagements von den zuständigen Instanzen (mit) gesteuert werden.

Beispiel - Unterbringung und Betreuung von Asylsuchenden und Flüchtlingen in der Schweiz

Gerade bei einer raschen Zunahme der Einreisen von Asylsuchenden und Flüchtlingen, wie sie im Jahr 2015 stattfand, ist die laufende Angebotsabstimmung sehr wichtig. Der Bund hat selber eine bestimmte Kapazität in den Empfangsstellen und weist dann die Asylsuchenden, proportional zur Einwohnerzahl, den Kantonen zu. Obwohl mit dieser Volatilität systemimmanent gerechnet werden muss, können grosse Unterschiede darin beobachtet werden, wie die einzelnen Kantone mit der Herausforderung umgehen. Während die einen kurzfristig neue Plätze bereitstellen können, sei es auch nur in Zivilschutzanlagen, scheinen andere mehr gefordert und stellen zur Not Zelte auf.

Hinweise auf die Umsetzung der Gestaltungsaufgabe 'Laufende Aktualisierung des Angebots' in den Anwendungsbeispielen

Übergabe der Behindertenhilfe vom Bund an die Kantone am Beispiel des Kantons Zug (Abschnitt 5.1):

$\mathrm{Zu}$ den Aufgaben der Angebotskoordination des Kantons gehören die Reaktion auf unerwartete Angebots- oder Nachfrageschwankungen, die kurzfristige Abstimmung der Angebote oder die Suche nach Angeboten für Einzelfälle von Menschen mit Behinderung, die besondere Bedürfnisse haben.

Überprüfung der BBIP im Kanton Zürich (Abschnitt 5.2):

Die Begleitevaluation der Pilotphase der Vermittlungs- und Triagestelle zeigte unter anderem, dass durch die zentrale Erfassung aller Anmeldungen durch diese Fachstelle die besten Voraussetzungen geschaffen wurden, um Zahlen für die laufende Bedarfserhebung zu erhalten. Im Weiteren erhielt die Triagestelle als zentrale Anlaufstelle für die Fragen der beruflichen und sozialen Integration 
im Asyl- und Flüchtlingsbereich vielfältige Hinweise über Probleme und Fragestellungen zu diesen Themen. Dabei wurde der Blick auch über die Grenzen der bestehen BBIP-Angebote hinaus erweitert und es konnten weiterführende Innovationen zur Fortentwicklung der Angebote angeregt werden.

\subsubsection{Gestaltungsaufgabe U3: Austauschprozesse im Versorgungssystem}

Wie im Abschnitt 3.3.2 in den Ausführungen zur Governance gezeigt wurde, stösst die sozialpolitische Steuerung an Grenzen, wenn versucht wird, vor allem über die Steuerungsmodi Macht und Hierarchie zu operieren. Ebenso werden die Grenzen sichtbar, wenn nur via Wettbewerb und Markt gesteuert wird. Die Systemgestaltung kann somit nicht nur durch politische Steuerung erfolgen, sondern sie muss aus einem Mix beziehungsweise einer Balance der Funktionslogiken und Werte der an einem Versorgungssystem beteiligten Akteure erfolgen. Die kollektive Findung der passenden Entscheidungsformen und damit die prozessuale Gestaltung eines Entwicklungsprozesses sind ebenso wichtig wie die inhaltlich-fachliche Bestimmung der Arbeit. Governance passiert dann, wenn die Unterschiedlichkeit der beteiligten Akteure anerkannt wird, wenn die verschiedenen Logiken aufeinander abgestimmt werden respektive wenn in diesem Sinn kooperativ und interaktiv gearbeitet wird. Um diesen Anspruch einzulösen, sind Kommunikation und Austausch im Versorgungsystem unter den verschiedenen Beteiligten die Voraussetzung. Dabei sind vielfältige Formen wie Jahrestreffen, Fachkonferenzen, EchoVeranstaltungen oder auch eigentliche Planungskonferenzen möglich. Die Kommunikation und die Kultur unter den Akteuren eines Versorgungssystems kann hier entscheidend gepflegt und entwickelt werden.

Beispiel - regelmässige Koordinationsgespräche der Verantwortlichen bei der Unterbringung und Betreuung von Asylsuchenden und Flüchtlingen in der Schweiz

Die Verantwortlichen von Bund und Kantonen treffen sich regelmässig, um die Unterbringung von Asylsuchenden zu koordinieren. Da es sich hier um ein politisch hochsensibles Thema handelt, ist eine ständige aktive und transparente Kommunikation unabdingbar. Die kantonalen Verantwortlichen pflegen den Austausch mit den Gemeindevertretungen vor Ort, dort wo die Menschen untergebracht sind. Teilweise wird auch der direkte Kontakt mit der Bevölkerung gesucht, zum Beispiel wenn es darum geht, ein neues Zentrum für Asylsuchende zu eröffnen. 
Hinweise auf die Umsetzung der Gestaltungsaufgabe 'Austausch im Versorgungssystem' in den Anwendungsbeispielen

Übergabe der Behindertenhilfe vom Bund an die Kantone am Beispiel des Kantons Zug (Abschnitt 5.1):

Gemäss Aussagen der Verantwortlichen hat sich im Kanton Zug eine Kultur etabliert, in welcher Kantonsverantwortliche und Einrichtungen im Sinn einer echten Partnerschaft auch ausserhalb der Aufsicht und begleitend zu den Leistungsvereinbarungen in ständigem, vertrauensbildendem Informationsaustausch und Dialog stehen.

Überprüfung der BBIP im Kanton Zürich (Abschnitt 5.2):

Die Anbietenden der BBIP-Programme beurteilten den Entwicklungsprozess insgesamt als sehr positiv. Die neue Konzeption wurde in vielerlei Hinsicht begrüsst: Die Verbindlichkeit und die Transparenz hätten sich deutlich erhöht. Der verstärkte Fokus auf Ziele und Zielformulierung hätten ganz allgemein zu einem zielorientierteren Handeln im Kontakt mit den Teilnehmenden geführt - ein Aspekt, der im Zusammenhang mit der neuen Konzeption sicher als besonders positiv und wertvoll zu beurteilen sei. Rückblickend lässt sich sagen, dass die Mitwirkung der Anbietenden ein wesentlicher Erfolgsfaktor für das Gelingen des Projekts war. Diese Einschätzung wurde von allen Beteiligten geteilt - und für das Team der Hochschule Luzern als externe Projektleitung war es eine zentrale Erfahrung, die das Handlungsverständnis für weitere Projekte prägte.

\subsubsection{Gestaltungsaufgabe U4: Reporting der Leistungserbringenden}

«Je komplizierter die Organisation, desto einfacher muss die Steuerung sein (...)» (Eisenreich/Halfar/Moss 2005: 96). Mit diesem Postulat führen die genannten Autoren in die Thematik des kennzahlengestützten Managements ein. Wenn es also um das Reporting der leistungserbringenden Einrichtung gegenüber der leistungsfinanzierenden, auftraggebenden Stelle geht, sind nicht möglichst viele und detaillierte Kennzahlen und Berichte zielführend, sondern es gilt, eine gezielte Auswahl zu treffen, die für die leistungserbringenden Organisationen zuverlässig bearbeitet und im Rahmen eines regelmässigen Berichtswesens zur Verfügung gestellt werden kann. Die Modalitäten dieser Berichterstattung werden in der Regel im Rahmen von Leistungsvereinbarungen festgelegt. In diesem Aushandlungsprozess ist sorgfältig zu erarbeiten, welche Informationen für die auftraggebende Stelle aus 
welchen Gründen wichtig sind. Im Abschnitt 3.3.6 zur Qualitätsentwicklung im Versorgungsystem werden in der dort gezeigten Abbildung zu den Ebenen der Qualitätsentwicklung verschiedene Steuerungslogiken sichtbar. Politik, Verwaltung, Management, Mitarbeitende und Leistungsadressatinnen und -adressaten haben, neben sich überschneidenden Kriterien, je eigene Bezugspunkte, um die Qualität einer Dienstleistung im Rahmen eines Versorgungssystems einzuschätzen. Diesen unterschiedlichen Bedürfnissen muss im Rahmen des Berichtswesens Rechnung getragen werden. Es ist ein kennzahlengestütztes Reportingmodell zu entwickeln, dass Informationen zur vereinbarten Leistung (fallbezogene Berichte und Kennzahlen), zu den Finanzen (betriebswirtschaftliche Berichte und Kennzahlen), zur Qualität (qualitätsbezogene Berichte und Kennzahlen), zu wesentlichen Entwicklungen im Versorgungssystem (strategische Berichte und Kennzahlen), je nach Gegenstand aber auch zu besonderen Risiken (risikobezogene Berichte und Kennzahlen) oder auch zu Immobilien (immobilienmanagementbezogene Berichte und Kennzahlen) enthalten kann. Auch Rhythmus und Form der Berichterstattung sind festzulegen. Bestimmte Informationen müssen möglicherweise monatlich, andere quartalsweise oder auch nur jährlich zur Verfügung gestellt werden.

Beispiel - Finanzierung der Unterbringung und Betreuung von Asylsuchenden und Flüchtlingen in der Schweiz

Die Finanzierung der Unterbringung und Betreuung von Asylsuchenden orientiert sich an der Kopfzahl der anwesenden Personen im jeweiligen Versorgungssystem. Dies gilt sowohl zwischen dem Bund und den Kantonen als auch zwischen den Kantonen und privaten Organisationen, die in einigen Kantonen Aufgaben im Auftrag der Kantone übernommen haben. Diese Kopfzahl steuert demzufolge die Ressourcen, ist aber auch gleichzeitig wegleitend für die Unterbringungskapazitäten, die bereitgestellt werden müssen. Berichte und Kennzahlen zu den Kopfzahlen, der Auslastung der Unterbringungsstrukturen, den Finanzen sowie der Umfeldentwicklung (politische Akzeptanz in Gemeinden, wo neu Asylsuchende platziert werden) sind deshalb für die Auftraggebenden sehr wichtig und müssen aufgrund ihrer hohen Volatilität mindestens monatlich bereitgestellt werden. 
Hinweise auf die Umsetzung der Gestaltungsaufgabe 'Reporting der leistungserbringenden Einrichtungen' in den Anwendungsbeispielen

Übergabe der Behindertenhilfe vom Bund an die Kantone am Beispiel des Kantons Zug (Abschnitt 5.1):

Die Leistungsvereinbarungen mit dem Kanton verpflichten die Einrichtungen, jährlich über zentrale Leistungs- und Finanzkennzahlen zu berichten.

Überprüfung der BBIP im Kanton Zürich (Abschnitt 5.2):

Ein erster Versuch zur Vereinheitlichung der Abrechnungen durch die Leistungserbringenden bestand darin, diese nach einem einheitlichen System durchzuführen. Eine Umfrage im Herbst 2009 bei allen Anbietenden zeigte jedoch, dass es bei den Anbietenden von Gemeinden nur mit grösstem Aufwand möglich wäre, die Rechnungslegung umzustellen, da diese an die Bedingungen der Staatsrechnung gebunden sind. Dies traf damals auch auf den grössten Anbieter zu, der mit zwölf Programmen rund 75\% der Kantonsbeiträge bezieht. Anstelle einer einheitlichen Rechnungslegung formulierten die befragten Programmanbietenden an ein Finanzcontrolling andere Erwartungen: Stetigkeit, Transparenz und ein vertretbarer Arbeitsaufwand zur Erstellung des Budgets und der Abrechnung. Als Fazit dieser Abklärungsphase wurde beschlossen, auf die Vorgabe einer spezifischen Rechnungslegungsform zu verzichten, jedoch für das Finanzcontrolling zwingend darauf zu achten, dass bei der Eingabe und dem Reporting (Jahresrechnung) durchgängig mit der gleichen Struktur der Formulare gearbeitet wird und bestimmte Kennzahlen vorgegeben werden. Unter Berücksichtigung der obengenannten Erwägungen hat das KSA Zürich auf Empfehlung der Hochschule Luzern im Frühling 2010 folgende Entscheide für die Weiterarbeit am Finanzmodell getroffen:

- $\quad$ In einer ersten Phase ist am bisherigen Modell der Programmfinanzierung festzuhalten.

- $\quad$ Für die Angebotstypen gemäss dem Konzept 2010 (Bürgisser/Mey 2011) sind detaillierte Leistungseinheiten zu definieren, die Bezug nehmen zu den inhaltlichen Vorgaben, wie sie im Qualitätsmanagement beschrieben sind (z.B. Anforderungen an einen eintägigen Kurs oder an ein Qualifikationsangebot). 
- $\quad$ Auf der Grundlage der definierten Leistungseinheiten sollen Kostenvergleiche erstellt werden.

- $\quad$ Auf der Basis des bisherigen Formulars ist ein einfaches Budget- und Abrechnungsformular mit wenigen Kostenarten und verständlichen Definitionen zu entwickeln.

\subsection{Gestaltungsphase 4: Weiterentwicklung (W)}

Beim letzten Arbeitsschritt im Arbeitskreislauf der Systemgestaltung handelt es sich um Weiterentwicklungsaufgaben. Im Unterschied zur laufenden Optimierung im Rahmen der Umsetzung von Zielen und Vorgaben (siehe Abschnitt 4.4) steht in dieser Phase eine grundsätzliche Überprüfung der Umweltbedingungen, Ziele, Angebote, Strukturen und der Leistungserbringenden im Zentrum. Möglicherweise erfahren durch die Systemgestaltung die betroffenen Versorgungssysteme oder die involvierten Einrichtungen umfangreichere Reformen. Den vier definierten Gestaltungsebenen folgend, werden folgende zentrale Aufgaben unterschieden:

(W1) Strategisches Controlling (Ebene 1: 'Programm und Finanzierung');

(W2) Angebotsüberprüfung (Ebene 2: 'Leistungen und Angebote');

(W3) Struktur- und Prozessevaluation (Ebene 3: 'Versorgungsstrukturen');

(W4) Weiterentwicklung der Leistungserbringenden (Ebene 4: 'Leistungserbringung').

Die folgende Abbildung gibt einen Überblick über die Aufgaben in der Phase der Weiterentwicklung. 


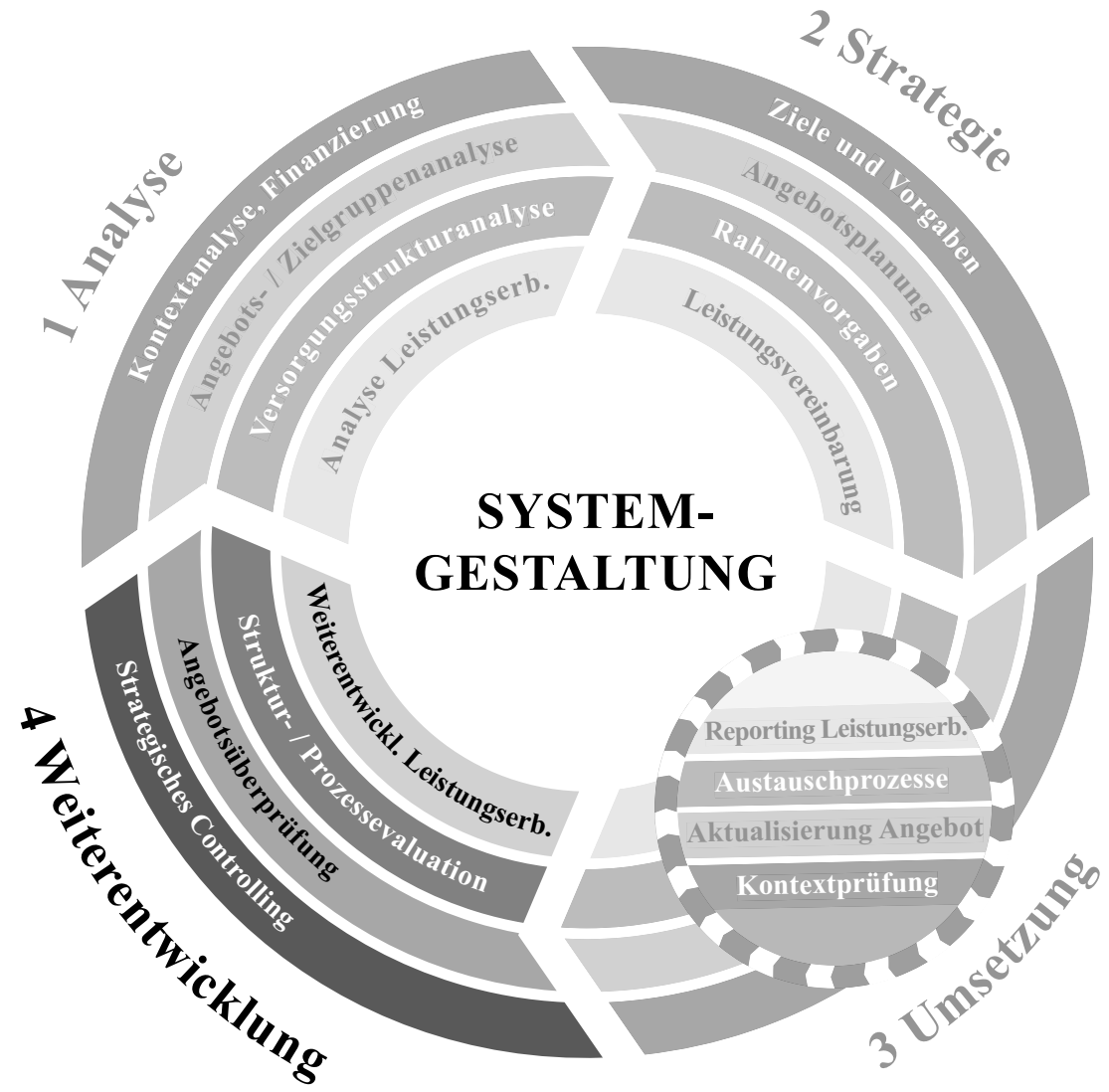

Abbildung 20: Weiterentwicklung in der Systemgestaltung: Phase 4 Quelle: eigene Darstellung

\subsubsection{Gestaltungsaufgabe W1: strategisches Controlling}

Strategisches Controlling hat die Funktion einer Steuerungsunterstützung. Georg Horcher nennt die Beschaffung, Aufbereitung und Analyse von Daten zur zielgerichteten Entscheidungsvorbereitung als Kernfunktion des Controllings (Grunwald/Horcher/Maelicke 2013: 217). Im strategischen Controlling steht die mittel- 
und langfristige Entwicklung im Fokus. Unter Berücksichtigung relevanter Umfeldentwicklungen wird die strategische Ausrichtung überprüft und allenfalls angepasst.

In periodischen Abständen wird im Rahmen eines strategischen Controllings überprüft, ob und in welcher Form die angestrebten übergeordneten Ziele erreicht werden. Die Ziele können sich dabei auf die angestrebte Angebots- und Versorgungslage, auf Wirkungen, Fragen der Wirtschaftlichkeit der Leistungserbringung, auf Versorgungsstrukturen oder eine ganze Reihe weiterer Aspekte beziehen. Dabei können einzelne Organisationen, Angebote oder Versorgungssysteme im Fokus stehen. Im Kontext von (sozial-)politischer Steuerung erfolgen strategische Controllings oft in Form von Rechenschaftsberichten. Nach Donat Knecht (2015) zeichnen sich klassische Rechenschaftsberichte durch die in der untenstehenden Abbildung aufgeführten Elemente aus.

\begin{tabular}{|l|l|}
\hline Berichtelement & Beschreibung \\
\hline Auftraggeber/-in & $\begin{array}{l}\text { Verfassungen oder Gemeindeordnungen regeln, dass Regierungen gegen- } \\
\text { über den Parlamenten über die Tätigkeit der Verwaltung Rechenschaft able- } \\
\text { gen müssen. }\end{array}$ \\
\hline Verfasser/-innen & $\begin{array}{l}\text { Verwaltungsbezogene Rechenschaftsberichte werden dezentral in den ver- } \\
\text { schiedenen Verwaltungseinheiten erstellt und zu einem Gesamtbericht zu- } \\
\text { sammengefügt. }\end{array}$ \\
\hline Fokus Fragestellung & $\begin{array}{l}\text { Der Schwerpunkt liegt üblicherweise bei den rechtlichen Grundlagen bezie- } \\
\text { hungsweise den darin definierten Zielen und öffentlichen Aufgaben und den } \\
\text { Legislaturzielen der Exekutive einerseits und den auf dieser Grundlage um- } \\
\text { gesetzten Massnahmen, den eingesetzten Ressourcen und dem Erreichungs- } \\
\text { grad der Ziele andererseits. Je nach Ausgestaltung der Verwaltungsorgani- } \\
\text { sation ist die Berichterstattung mehr oder weniger standardisiert, kosten- } \\
\text { transparent und outputorientiert. }\end{array}$ \\
\hline Methodik & $\begin{array}{l}\text { Die Berichterstattung ist ein routinemässiger Verwaltungsvorgang. Dieser } \\
\text { besteht in der Identifikation des gesetzlichen Auftrages und der politischen } \\
\text { Zielsetzung, der Definition eines einheitlichen Berichterstattungsrasters für } \\
\text { die einzelnen Verwaltungseinheiten, einer dezentralen Berichterstattung der } \\
\text { innerhalb dieser Einheiten Zuständigen sowie in der Gesamtbeurteilung und } \\
\text { der Verabschiedung des Berichts durch die Exekutive. }\end{array}$ \\
\hline Datengrundlagen & $\begin{array}{l}\text { Rechenschaftsberichte bedienen sich verschiedener interner und amtlicher } \\
\text { Statistiken, der Zahlen der Staatsrechnung und Selbstbeschreibungen in } \\
\text { Prosa. }\end{array}$ \\
\hline Ergebnis & $\begin{array}{l}\text { Rechenschaftsberichte werden in der Regel nicht von unabhängiger Seite er- } \\
\text { stellt und sind häufig politisch gefärbt. Mögliche Erkenntnisse aus Rechen- } \\
\text { schaftsberichten sind im besten Fall: von der Verwaltung erbrachte Leistun- } \\
\text { gen, Kosten der erbrachten Leistungen, Wirkungen der erbrachten Leistungen } \\
\text { und Erreichungsgrad der gesetzten Ziele. }\end{array}$ \\
\hline
\end{tabular}

Abbildung 21: Elemente eines klassischen Rechenschaftsberichts Quelle: Knecht (2015: 21) 


\section{Beispiel - Auszug aus dem Geschäftsbericht 2015 des Kantons Zug}

\section{Grundauftrag}

Das Sozialamt entwickelt Lösungen für soziale Brennpunkte und unterstützt mit seinen Dienstleistungen den gesellschaftlichen Zusammenhalt der Zuger Bevölkerung. Es ist zuständig für die Planung, Steuerung und Aufsicht sowie die Information und Koordination wichtiger sozial- und gesellschaftspolitischer Massnahmen, insbesondere in den Bereichen Asyl, soziale Einrichtungen, Sozialhilfe, Integration, Kind, Jugend, Familie und Alter.

2 Zielsetzungen und Erfolgskontrolle

1, 2, 3 = Wiederkehrende Ziele

A, B, C = Projekte

Erreicht

$L 1, L 2, L 3=$ Legislaturziele

Teilweise erreicht

Nr. Zielsetzungen

Indikatoren und Zielgrössen 2015

Nicht erreicht

$\frac{\text { Leistungsgruppe 1: Soziale Dienste Asyl }}{\text { Regelmässige Beratungen }}$
$\frac{\text { Gezielte Förderung der Integration }}{3}$
$\frac{\text { Ersatz wegfallender Asyl- und Nothilfe- }}{\text { unterkünfte }}$
$\begin{aligned} & \text { Freiwilligenarbeit ausbauen und } \\ & \text { konsolidieren }\end{aligned}$

Anzahl Beratungsgespräche / Monat

im Verhältnis zur Zielgruppe: $70 \%$

(315 Gespräche / 450 Personen)

Anzahl Leistungsempfangende mit

1 beruflichen Integrationsmassnahme:

$40 \%$ (ca. 200 Personen / 80 Massnahmen);

Anzahl Leistungsempfangende mit

1 sprachlichen Integrationsmassnahme:

$40 \%$ (ca. 200 Personen / 80 Massnahmen)

$100 \%$ genügend Unterkunftsplätze gemäss Fallzahlenentwicklung

Mit neuen Freiwilligen (ca. 5) Eintrittsgespräch führen

Mit allen Freiwilligen (ca. 15)

- Vereinbarung abschliessen

- Begleitungsgespräch 2 x pro Jahr

Leistungsgruppe 2: Steuerung und Finanzierung von sozialen Organisationen

6 Kontinuierliches und systematisches Kontraktmanagement (Überprüfung Angebot, Qualität und Finanzen, Auszahlungen, Anpassungen der Leistungs-

Anzahl Controllinggespräche: 17: Anzahl Neuverhandlungen von Vereinbarungen: 4 und Subventionsvereinbarungen)

7 Regelmässige Überprüfung der Bewilligungs- und Anerkennungsvoraussetzungen

Anzahl Überprüfungen: 15

A $\quad \begin{aligned} & \text { Einstufungssystem IBB (Individueller } \\ & \text { Betreungsbedarf): Einführung Indikatoren- } \\ & \text { raster und Pilotphase }\end{aligned}$
$\begin{aligned} & \text { Aufarbeitung Fürsorgerische Zwangsmass- Schlussbericht liegt vor } \\ & \text { nahmen im Kanton Zug (bis 1981) }\end{aligned}$

Abbildung 22: Ziele und deren Umsetzungsstand beim Sozialamt Quelle: Kanton Zug (2015: 100)
16 Controllinggespräche, 1 geplante Subventionsvereinbarung (SV) nicht abgeschlossen; 2 Neuverhandlungen, aufgrund Entlastungsprogramms 1 Leistungsvereinbarung nur verlängert, 1 SV nicht mehr abgeschlossen

14 Überprüfungen; es werden auch ausserkantonale Nicht-IVSE Einrichtungen geprüft, um Kostenübernahmegarantien sprechen zu können; Anzahl und Aufwand war überdurchschnittlich hoch, eine regelmässige Prüfung wurde verschoben

Aufgrund fehlender Finanzierungsmöglichkeiten konnte Bericht noch nicht in Auftrag gegeben werden 
Hinweis auf die Umsetzung der Gestaltungsaufgabe 'strategisches Controlling' in den Anwendungsbeispielen

Übergabe der Behindertenhilfe vom Bund an die Kantone am Beispiel des Kantons Zug (Abschnitt 5.1):

Bevor im Kanton Zug eine neue Planungsperiode in Angriff genommen wird, ist $\mathrm{zu}$ prüfen, wie weit die (sozialpolitischen) Vorgaben beziehungsweise Grundsätze und Ziele in der vergangenen Periode umgesetzt werden konnten. Dabei ist vor allem die grundsätzliche Stossrichtung zu beleuchten, sind die formulierten Ziele doch eher langfristiger Natur und können kaum innerhalb einer dreijährigen Planungsperiode vollständig erreicht werden.

\subsubsection{Gestaltungsaufgabe W2: Angebotsüberprüfung}

Die Überprüfung von Dienstleistungen und Angeboten in der Sozialwirtschaft erfolgt - vergleichbar mit anderen Politikbereichen - oft in Form von Evaluationen. Im Zentrum stehen dabei Vollzugs- und Wirkungsevaluationen. Gegenstand von Vollzugsevaluationen ist die Art und Weise, wie Dienstleistungen erbracht werden (Widmer/Bewyl/Fabian 2009). Im Zentrum stehen hier Prozesse, Abläufe oder Vorgehensweisen der Angebotserstellung oder der Leistungserbringung. Vollzugsevaluationen fokussieren beispielsweise Angemessenheit und Effizienz der Leistungserbringung. Im Zentrum von Wirkungsevaluationen steht die Frage, ob die spezifischen Dienstleistungen oder Angebote die intendierten Ziele erreichen. Im Rahmen von Wirkungsevaluationen wird zwischen Output, Outcome und Impact unterschieden. Der unmittelbare Output bezeichnet die für die Zielgruppen oder Nutzer/-innen direkt erbrachten Ergebnisse. Outcomes beziehen sich dagegen auf die eigentlichen Wirkungen, die mit den Dienstleistungen erzielt werden, wie zum Beispiel Verhaltensänderungen von Zielgruppen oder Änderungen in der Praxis von Fachpersonen. Schliesslich können auch Wirkungen auf der Systemebene untersucht werden; in diesem Fall wird von Impact gesprochen. Im Fokus stehen hier generelle gesellschaftliche Auswirkungen von sozialen Dienstleistungen oder Angeboten wie zum Beispiel die Erhöhung der sozialen Integration von Sozialhilfebezügerinnen und Sozialhilfebezügern oder ein volkswirtschaftlicher Nutzen in Form von gesamtgesellschaftlichen Kosteneinsparungen, die aus einer verbesserten sozialen Integration resultieren. 


\section{Beispiel - Begleitevaluation des ersten $Q D Z$ in Schaffhausen}

Bei der Evaluation durch das Wohnforum der Eidgenössischen Technischen Hochschule ETH Zürich (Hugentobler/Wurster 2013), die sich von Januar 2011 bis Juni 2013 erstreckte, stand die Begleitung der ersten zwei Betriebsjahre des Pilotprojekts «Quartierdienstleistungszentrum» im Zentrum. Untersucht wurde, in welchem Ausmass und von wem die angebotenen Hol- und Bring-Dienstleistungen genutzt und wie sie eingeschätzt werden.

Die Evaluation der Aufbau- und frühen Entwicklungsphase des QDZ zeigt, dass sich das erste Zentrum, unter Überwindung verschiedener räumlich-struktureller Unzulänglichkeiten und organisatorischer Hindernisse, als Konzept und Betrieb etabliert, neue Impulse geschaffen und bereits viel erreicht hat. Wichtige Beiträge liegen in der Erweiterung des quartiernahen Dienstleistungsangebots, der stärkeren Vernetzung von professionellen Dienstleistenden, Quartierakteuren sowie in der Schaffung von Strukturen, welche die Zielsetzung «ambulant vor stationär» unterstützen. Die verschiedenen, bereits seit dem Bezug des Neubaus bestehenden Wohnformen des QDZ sind nach wie vor gut nachgefragt und weisen eine hohe Auslastung auf. Der grosse Mehrzweckraum erfreut sich starker Nachfrage und wurde in den letzten zwei Jahren jeweils über 300-mal benutzt, wovon zwei Drittel der Nutzung auf externe Gruppierungen entfielen. Auch das Gastronomieangebot ist attraktiv; die Nachfrage hat in den letzten zwei Jahren stetig zugenommen. Unter den ambulanten Angeboten des QDZ erbringt das ambulante Pflegeteam vor Ort die wichtigste Dienstleistung. Die Integration der ambulanten Pflege im Zentrum ermöglicht eine engere Verschränkung und vermehrte Zusammenarbeit zwischen ambulanter und stationärer Pflege. Auf diese Weise fördern die räumliche Nähe und der Austausch zwischen dem ambulanten Pflegeteam, der Heimleitung und weiteren Anbietenden vor Ort (Physiotherapie oder Sozialberatung) angepasste und aufeinander abgestimmte Lösungen und ein rasches Handeln bei bevorstehenden oder unerwarteten Heimeintritten. Gleichzeitig hat die ambulante Pflege eine wichtige Informations- und Vermittlungsfunktion bezüglich der $\mathrm{Zu}$ sammenarbeit verschiedener professioneller Dienstleistender und in Bezug auf die weiteren Angebote des QDZ.

\subsubsection{Gestaltungsaufgabe W3: Struktur- und Prozessevaluation}

Im Rahmen von Vollzugsevaluationen werden häufig die Strukturen und Prozesse der Dienstleistungserbringung einer systematischen Betrachtung und Bewertung unterzogen. Diese Evaluationen der Leistungserbringung in Versorgungssystemen 
fokussieren ähnliche Fragen wie sie bereits bei der Analyse von Versorgungsstrukturen beschrieben wurden. Im Zentrum stehen Fragen zu folgenden Strukturaspekten:

- Zusammenarbeit der relevanten Akteure innerhalb des Versorgungssystems: Wie gut funktioniert die Zusammenarbeit zwischen den Einrichtungen?

- Angebotsabstimmung: Sind die Angebote der Einrichtungen in der Versorgungskette zweckmässig aufeinander abgestimmt (keine Doppelspurigkeiten oder Lücken) und hinreichend durchlässig?

- Zugang zum Angebot / Erreichbarkeit des Angebots: Werden die anvisierten Zielgruppen mit dem Dienstleistungsangebot überhaupt erreicht?

- Vermittlung / Zuweisung: Erfolgt die Zuweisung zum Angebot strukturiert und nach zweckmässigen Kriterien, sodass eine optimale Passung zwischen dem Zielgruppenprofil und dem Angebotsprofil erreicht werden kann?

- Räumliche Gestaltung: Sind die Grösse des Einzugsgebiets, die Lage der leistungserbringenden Organisationen im Raum (Erreichbarkeit), deren Spezialisierungsgrad und deren Grösse zweckmässig gestaltet?

Während Wirkungsevaluationen oft als sogenannte summative Evaluationen durchgeführt werden, sind Struktur- und Prozessevaluationen häufig als sogenannte formative Evaluationen konzipiert. Summative Evaluationen ziehen beispielsweise am Ende eines Pilotversuchs eine Bilanz, ob und inwiefern die angestrebten Wirkungen erreicht wurden. Sie nehmen zu einem bestimmten Zeitpunkt eine Bewertung vor, die einen bilanzierenden Generalisierungsanspruch ausweist. Formative Evaluationen werden häufig zur fortlaufenden Verbesserung von Dienstleistungen und Angeboten verwendet. Ihr Fokus liegt weniger auf einer Bilanzierung, sondern auf der fortlaufenden Optimierung.

Beispiel - Grenzen institutioneller Angebotsstrukturen bei zurückgezogen lebenden älteren Menschen

Ein Forschungsteam der Technischen Hochschule Köln hat die Lebenssituation desjenigen Teils der älteren Bevölkerung genauer untersucht, der zurückgezogen lebt, nur eingeschränkt in die sozialräumliche Umgebung eingebunden ist und daher kaum von den bislang zur Verfügung stehenden Versorgungsstrukturen erreicht werden kann (Schubert/Leitner/Schönbrodt/Veil/Vukoman 2014). In weiteren Schritten wurden die Aktionsräume und die Beziehungsgelegenheiten dieser zurückgezogen lebenden älteren Menschen in ausgewählten Wohnquartieren der Stadt Köln näher erforscht. Parallel dazu wurden bestehende Projekte in Deutsch- 
land und in Nachbarstaaten erhoben, die das Ziel der gesellschaftlichen Einbindung zurückgezogen lebender älterer Menschen verfolgen. Auf der Grundlage der dabei gewonnenen Erkenntnisse wurde ein neues Infrastrukturkonzept zur Stärkung derjenigen älteren Menschen entwickelt, die abgeschieden leben. Das neue Konzept fokussiert alltäglich zugängliche Orte und Begegnungsmöglichkeiten im jeweiligen Wohnquartier, die eine Verbindung zwischen institutionalisierten und informellen Formen der Unterstützung und Hilfe fördern und auf diese Weise mehr Teilhabechancen für zurückgezogen lebende ältere Menschen eröffnen.

Hinweis auf die Umsetzung der Gestaltungsaufgabe 'Überprüfung und Weiterentwicklung von Strukturen und Prozessen' in den Anwendungsbeispielen

Überprüfung der BBIP im Kanton Zürich (Abschnitt 5.2):

Die Begleitevaluation der Vermittlungs- und Triagestelle fokussierte Fragen zum Nutzen und zur Funktionalität dieser Fachstelle. Um diese beiden Aspekte zu untersuchen, wurden Verlaufsdaten ausgewertet sowie die mit dem Aufbau der Triagestelle betrauten Fachpersonen und Vertreter/-innen der verschiedenen Anspruchsgruppen - vor allem fallführende respektive zuweisende Stellen und Programmanbietende - befragt. Aus den Befragungen der Vertreter/-innen (30 Personen) der verschiedenen Anspruchsgruppen, der fallführenden Stellen und der Programmanbietenden sowie der mit der Umsetzung des Projekts beauftragen Fachpersonen der Triagestelle liessen sich wesentliche Erkenntnisse für die Weiterentwicklung der Fachstelle gewinnen.

\subsubsection{Gestaltungsaufgabe W4: Weiterentwicklung Leistungserbringender}

Bei der Weiterentwicklung der einzelnen leistungserbringenden Organisation handelt es sich nicht um regelmässige Anpassungen der Anzahl der Dienstleistungen wie zum Beispiel Wohnplätze für Menschen mit Behinderung oder Justierungen beim Qualitätsentwicklungssystem. Eine Weiterentwicklung liegt dann vor, wenn substanzielle Anpassungen auf der Ebene der Dienstleistungen oder bei den Strukturen einer Einrichtung vorgenommen werden (müssen). Dies ist beispielsweise der Fall, wenn ein ganzes Dienstleistungspaket neu aufgebaut oder gestrichen werden soll. Auslöser für Weiterentwicklungsprozesse der Leistungserbringenden könnten unter anderem die Folgenden sein:

- $\quad$ die (Neu-)Vergabe von Leistungsaufträgen;

- $\quad$ der Wegfall eines Leistungsauftrags; 
- $\quad$ eine Überprüfung auf der strategischen Ebene;

- Veränderungen sozialpolitischer Vorgaben oder Rahmenbedingungen;

- die Konfrontation mit neuen sozialen Problemen;

- $\quad$ Erkenntnisse aus den regelmässigen Reportings der Einrichtungen;

- Ergebnisse einer Vollzugs- oder Wirkungsevaluation;

- $\quad$ ein Wechsel der Trägerschaft.

\section{Beispiel - Überprüfung der Angebotspalette von Alters- und Pflegeheimen}

Angesichts der demografischen Alterung, der Wünsche und Anliegen älterer Menschen, so lange wie möglich zu Hause leben zu können sowie der Entwicklungen im Gesundheitswesen und im Bereich der ambulanten Angebote stehen Altersund Pflegeheime vor grossen Herausforderungen. Diese aktuellen Veränderungen nehmen zurzeit Auftraggebende oder Trägerschaften zum Anlass, ihre bisherige auf Pflege und Betreuung ausgerichtete Angebotspalette zu überprüfen. Im Zentrum steht dabei häufig eine Diversifizierung des Angebots. So wird beispielsweise erwogen, selbstständiges Wohnen mit abrufbaren Dienstleistungen anzubieten, sich auf spezifische Angebote (z.B. Pflege von Menschen mit einer Demenzerkrankung oder Palliativpflege) auszurichten oder ambulante quartiernahe Angebote in den Betrieb zu integrieren.

Hinweis auf die Umsetzung der Gestaltungsaufgabe 'Weiterentwicklung der Leistungserbringenden' in den Anwendungsbeispielen

Überprüfung der BBIP im Kanton Zürich (Abschnitt 5.2):

Häufig werden soziale Einrichtungen am Ende eines Pilotprojekts oder einer Pilotphase einer Evaluation und Weiterentwicklung unterzogen. Ein Beispiel dafür stellt die im nächsten Kapitel beschriebene Begleitevaluation der Vermittlungs- und Triagestelle im Kanton Zürich im Rahmen der BBIP dar. Die im Abschnitt 4.5.2 beschriebene Evaluation der erstmaligen Realisierung eines QDZ in der Stadt Schaffhausen kann als weiteres Beispiel gelten. 
Open Access Dieses Kapitel wird unter der Creative Commons Namensnennung 4.0 International Lizenz (http://creativecommons.org/licenses/by/4.0/deed.de) veröffentlicht, welche die Nutzung, Vervielfältigung, Bearbeitung, Verbreitung und Wiedergabe in jeglichem Medium und Format erlaubt, sofern Sie den/die ursprünglichen Autor(en) und die Quelle ordnungsgemäß nennen, einen Link zur Creative Commons Lizenz beifügen und angeben, ob Änderungen vorgenommen wurden.

Die in diesem Kapitel enthaltenen Bilder und sonstiges Drittmaterial unterliegen ebenfalls der genannten Creative Commons Lizenz, sofern sich aus der Abbildungslegende nichts anderes ergibt. Sofern das betreffende Material nicht unter der genannten Creative Commons Lizenz steht und die betreffende Handlung nicht nach gesetzlichen Vorschriften erlaubt ist, ist für die oben aufgeführten Weiterverwendungen des Materials die Einwilligung des jeweiligen Rechteinhabers einzuholen.

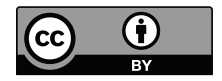

